\title{
LAPLACIAN OPERATORS AND RADON TRANSFORMS ON GRASSMANN GRAPHS
}

\author{
JOSÉ MANUEL MARCO AND JAVIER PARCET
}

\begin{abstract}
Let $\Omega$ be a vector space over a finite field with $q$ elements. Let $\mathrm{G}$ denote the general linear group of endomorphisms of $\Omega$ and let us consider the left regular representation $\rho: \mathrm{G} \rightarrow \mathcal{B}\left(L_{2}(\mathrm{X})\right)$ associated to the natural action of $\mathrm{G}$ on the set $\mathrm{X}$ of linear subspaces of $\Omega$. In this paper we study a natural basis $\mathbf{B}$ of the algebra $\operatorname{End}_{\mathrm{G}}\left(L_{2}(\mathrm{X})\right)$ of intertwining maps on $L_{2}(\mathrm{X})$. By using a Laplacian operator on Grassmann graphs, we identify the kernels in $\mathbf{B}$ as solutions of a basic hypergeometric difference equation. This provides two expressions for these kernels. One in terms of the $q$-Hahn polynomials and the other by means of a Rodrigues type formula. Finally, we obtain a useful product formula for the mappings in $\mathbf{B}$. We give two different proofs. One uses the theory of classical hypergeometric polynomials and the other is supported by a characterization of spherical functions in finite symmetric spaces. Both proofs require the use of certain associated Radon transforms.
\end{abstract}

\section{INTRODUCTION}

Let $\mathrm{X}$ be a homogeneous space with respect to a given finite group $\mathrm{G}$. That is, the group $\mathrm{G}$ acts transitively on the set $\mathrm{X}$. Then, we can consider the left regular representation $\rho: \mathrm{G} \rightarrow \mathcal{B}\left(\mathrm{V}_{\mathrm{X}}\right)$ of $\mathrm{G}$ into the Hilbert space $\mathrm{V}_{\mathrm{X}}$ of complex-valued functions $\varphi: X \rightarrow \mathbb{C}$. In this context, it is well-known that the algebra $\operatorname{End}_{\mathrm{G}}\left(\mathrm{V}_{\mathrm{X}}\right)$ of intertwining operators with respect to $\rho$ codify some relevant information. For instance, $\operatorname{End}_{\mathrm{G}}\left(\mathrm{V}_{\mathrm{X}}\right)$ is abelian if and only if the left regular representation $\rho$ is multiplicity-free. In this case, following Terras' book [11, we say that $\mathrm{X}$ is a finite symmetric space with respect to $G$. When dealing with finite symmetric spaces, any explicit expression for the kernels of the orthogonal projections onto the irreducible components of $\mathrm{V}_{\mathrm{X}}$ is interesting. Indeed, the main motivation lies in the fact that these expressions can be usually regarded as combinatorial versions of the irreducible characters. More generally, if $\mathrm{X}$ and $\mathrm{Y}$ are finite symmetric spaces with respect to $\mathrm{G}$, some information about the relations between $\mathrm{X}$ and $\mathrm{Y}$ in the group theory level can be obtained by studying the space $\operatorname{Hom}_{\mathrm{G}}\left(\mathrm{V}_{\mathrm{X}}, \mathrm{V}_{\mathrm{Y}}\right)$ of intertwining homomorphisms from $V_{X}$ to $V_{Y}$. For instance, the dimension of the space $\operatorname{Hom}_{\mathrm{G}}\left(\mathrm{V}_{\mathrm{X}}, \mathrm{V}_{\mathrm{Y}}\right)$ coincides with the number of irreducible components that $\mathrm{V}_{\mathrm{X}}$ and $\mathrm{V}_{\mathrm{Y}}$ have in common.

Two natural problems arise in this setting. In order to state them, let us consider the Radon transform $\mathcal{R}_{\mathcal{Z}}: \mathrm{V}_{\mathrm{X}} \rightarrow \mathrm{V}_{\mathrm{Y}}$ associated to a G-invariant subset $\mathcal{Z} \subset \mathrm{X} \times \mathrm{Y}$

$$
\mathcal{R}_{\mathcal{Z}} \varphi(y)=\sum_{x:(x, y) \in \mathcal{Z}} \varphi(x)
$$

Partially supported by the Project MTM2004-00678, Spain.

2000 Mathematics Subject Classification: Primary 05A30, 05E30. Secondary 20G40, 33D45.

Key words and phrases: Symmetric space, Difference equation, Basic hypergeometric function. 
Taking the G-invariant subset $\mathcal{Z}$ to be each of the orbits $\mathcal{O}_{1}, \mathcal{O}_{2}, \ldots, \mathcal{O}_{d}$ of the action of $\mathrm{G}$ on the product $\mathrm{X} \times \mathrm{Y}$, we obtain a basis of the space $\operatorname{Hom}_{\mathrm{G}}\left(\mathrm{V}_{\mathrm{X}}, \mathrm{V}_{\mathrm{Y}}\right)$ made up of Radon transforms

$$
\mathbf{B}_{1}=\left\{\mathcal{R}_{1}, \mathcal{R}_{2}, \ldots, \mathcal{R}_{d}\right\}
$$

On the other hand, since the dimension of $\operatorname{Hom}_{\mathrm{G}}\left(\mathrm{V}_{\mathrm{X}}, \mathrm{V}_{\mathrm{Y}}\right)$ coincides of the number of irreducible components that $\mathrm{V}_{\mathrm{X}}$ and $\mathrm{V}_{\mathrm{Y}}$ have in common, we obtain the following orthogonal decompositions

$$
\begin{aligned}
& \mathrm{V}_{\mathrm{X}}=\mathrm{W}_{\mathrm{X}} \oplus \bigoplus_{1 \leq s \leq d} \mathrm{~V}_{\mathrm{X}, s}, \\
& \mathrm{~V}_{\mathrm{Y}}=\mathrm{W}_{\mathrm{Y}} \oplus \bigoplus_{1 \leq s \leq d} \mathrm{~V}_{\mathrm{Y}, s},
\end{aligned}
$$

where $\mathrm{V}_{\mathrm{X}, s_{1}}$ is equivalent to $\mathrm{V}_{\mathrm{Y}, s_{2}}$ if and only if $s_{1}=s_{2}$. Then we introduce non-zero operators $\Lambda_{s} \in \operatorname{Hom}_{\mathrm{G}}\left(\mathrm{V}_{\mathrm{X}, s}, \mathrm{~V}_{\mathrm{Y}, s}\right)$ and we regard them as elements of $\operatorname{Hom}_{\mathrm{G}}\left(\mathrm{V}_{\mathrm{X}}, \mathrm{V}_{\mathrm{Y}}\right)$ vanishing on $\mathrm{V}_{\mathrm{X}} \ominus \mathrm{V}_{\mathrm{X}, s}$. Each mapping $\Lambda_{s}$ is an intertwining isomorphism between $\mathrm{V}_{\mathrm{X}, s}$ and $\mathrm{V}_{\mathrm{Y}, s}$ and, by Schur lemma, it is unique up to a constant factor. This family of mappings provide another basis of $\operatorname{Hom}_{G}\left(V_{X}, V_{Y}\right)$

$$
\mathbf{B}_{2}=\left\{\Lambda_{1}, \Lambda_{2}, \ldots, \Lambda_{d}\right\} .
$$

Both bases $\mathbf{B}_{1}$ and $\mathbf{B}_{2}$ are orthogonal with respect to the Hilbert-Schmidt inner product. The first problem we are interested on is to obtain the coefficients relating the bases described above. On the other hand, given three finite symmetric spaces $\mathrm{X}_{1}, \mathrm{X}_{2}$ and $\mathrm{X}_{3}$, operator composition provides a bilinear mapping

$$
\operatorname{Hom}_{\mathrm{G}}\left(\mathrm{V}_{\mathrm{X}_{2}}, \mathrm{~V}_{\mathrm{X}_{3}}\right) \times \operatorname{Hom}_{\mathrm{G}}\left(\mathrm{V}_{\mathrm{X}_{1}}, \mathrm{~V}_{\mathrm{X}_{2}}\right) \longrightarrow \operatorname{Hom}_{\mathrm{G}}\left(\mathrm{V}_{\mathrm{X}_{1}}, \mathrm{~V}_{\mathrm{X}_{3}}\right)
$$

given by

$$
\left(\Lambda^{2,3}, \Lambda^{1,2}\right) \mapsto \Lambda^{2,3} \circ \Lambda^{1,2} .
$$

The second problem we want to study is to write the products $\Lambda_{s}^{2,3} \circ \Lambda_{s}^{1,2}$ in terms of the operators $\Lambda_{s}^{1,3}$. In this paper we solve the problems presented above for the Grassmann graphs associated to the general linear group over a finite field. For the first problem, we meet a large family of $q$-Hahn polynomials and we obtain in this way a combinatorial interpretation of this family of classical hypergeometric polynomials. Besides, we provide a Rodrigues type formula for these polynomials adapted to the present framework. For the second problem, we obtain the product formula by two different processes. One lies in the theory of classical hypergeometric polynomials and the other in the theory of finite symmetric spaces and spherical functions. The second approach also provides certain identities for the kernels in $\mathbf{B}_{2}$ which might be of independent interest. The results we present in this paper constitute the $q$-analogue of those given in 8 . The main idea is to identify certain Laplacian type operators on Grassmann graphs as hypergeometric type operators. This procedure will allow us to apply some results on classical hypergeometric polynomials which have appeared recently in 9. The paper 9] provides a new approach to the theory of classical hypergeometric polynomials which somehow lives between the theories developed by Askey and Wilson 2] on one side and by Nikiforov, Suslov and Uvarov [10] on the other. One of the main motivations for this paper is to show the efficiency of the point of view suggested in $[9]$. 
The organization of the paper is as follows. Let $\Omega$ be a finite-dimensional vector space over a finite field $\mathbb{K}$ and let $G$ be the general linear group of endomorphisms of $\Omega$. Let us consider the left regular representation $\rho: \mathrm{G} \rightarrow \mathcal{B}\left(\mathrm{V}_{\mathrm{X}}\right)$ associated with the natural action of $\mathrm{G}$ on the set $\mathrm{X}$ of linear subspaces of $\Omega$. Section 1 is devoted to describe a natural basis $\mathbf{B}$ of the intertwining algebra $\operatorname{End}_{G}\left(V_{X}\right)$. The Laplacian operators on Grassmann graphs are studied in Section 2 This is used in Section 3 to identify these operators with some well-known hypergeometric type operators. Then we provide polynomic expressions and Rodrigues type formulas for the kernels of the operators in B. Finally, in Sections 4 and 5 we give two different proofs of the product formula mentioned above. Section 4 uses the theory of classical hypergeometric polynomials while the proof given in Section 5 lies in a characterization of spherical functions on finite symmetric spaces.

After having written this paper, the authors were informed on the existence of Dunkl's paper [5], which also identifies the kernels mentioned above as $q$-Hahn polynomials and studies similar relations for them. We note however that there also exist some significant differences between both papers. Indeed, our main product formula is not obtained in [5] while the Rodrigues formulas deduced from [9] (with non-ramified weights) are new.

\section{The object to STUdy}

Let $\mathbb{K}$ denote the field $\mathbb{F}_{q}$ with $q$ elements for some power of a prime $q$. In what follows, $\Omega$ will be a finite-dimensional vector space over $\mathbb{K}$. If $n$ stands for $\operatorname{dim} \Omega$, we shall also consider the set X of linear subspaces of $\Omega$ and the sets $\mathrm{X}_{r}$ of $r$-dimensional subspaces of $\Omega$ for $0 \leq r \leq n$. For any $(x, y) \in \mathrm{X} \times \mathrm{X}$, we define

$$
\begin{aligned}
& \partial(x, y)=\operatorname{dim}(x /(x \cap y)), \\
& \bar{\partial}(x, y)=\partial(x, y)+\partial(y, x) .
\end{aligned}
$$

Recall that, by Grassmann formula, we also have $\partial(x, y)=\operatorname{dim}((x+y) / y)$. Taking $\partial(x)=\operatorname{dim}(x)$, we can write $\bar{\partial}(x, y)=\partial(x)+\partial(y)-2 \partial(x \cap y)$. The function $\bar{\partial}: \mathrm{X} \times \mathrm{X} \rightarrow \mathbb{R}_{+}$is clearly a graph distance on $\mathrm{X}$ and the same happens with the restriction $\partial_{r}: \mathrm{X}_{r} \times \mathrm{X}_{r} \rightarrow \mathbb{R}_{+}$of $\partial$ on each $\mathrm{X}_{r}$. The distance $\partial_{r}$ imposes on $\mathrm{X}_{r}$ an structure of distance-regular graph. These graphs are well-known in the literature as Grassmann graphs, see 4 for more on this. Besides, we shall also need to consider the vector space $\mathrm{V}$ of complex valued functions $\varphi: \mathrm{X} \rightarrow \mathbb{C}$ and its subspaces $\mathrm{V}_{r}$ made up of functions $\varphi: \mathrm{X}_{r} \rightarrow \mathbb{C}$ for $0 \leq r \leq n$. Notice that both $\mathrm{V}$ and $\mathrm{V}_{r}$ are vector spaces over the complex field. If we consider the natural Hilbert space structure on these spaces, so that $\mathrm{V}=L_{2}(\mathrm{X})$ and $\mathrm{V}_{r}=L_{2}\left(\mathrm{X}_{r}\right)$, we clearly have the orthogonal decomposition

$$
\mathrm{V}=\bigoplus_{r=0}^{n} \mathrm{~V}_{r}
$$

1.1. Finite symmetric spaces. Before stating in detail the problem we want to study, we give a brief summary of results on finite symmetric spaces and spherical functions that will be used in the sequel. For further information on these topics see [11 and the references cited there. Let $G$ be a finite group acting on a finite set $\mathrm{X}$. This action gives rise to the left regular representation $\rho: \mathrm{G} \rightarrow \mathcal{B}\left(L_{2}(\mathrm{X})\right)$, defined as follows

$$
(\rho(g) \varphi)(x)=\varphi\left(g^{-1} x\right) .
$$


Assume that the action $\mathrm{G} \times \mathrm{X} \rightarrow \mathrm{X}$ is transitive, so that $\mathrm{X}$ becomes a homogeneous space. Then $\mathrm{X}$ is called a finite symmetric space with respect to the group $\mathrm{G}$ if the algebra $\operatorname{End}_{\mathrm{G}}\left(L_{2}(\mathrm{X})\right)$ of intertwining endomorphisms of $L_{2}(\mathrm{X})$ is abelian.

Remark 1.1. $\operatorname{End}_{\mathrm{G}}\left(L_{2}(\mathrm{X})\right)$ is abelian if and only if $\rho$ is multiplicity-free, see [11.

Now, if we are given two finite symmetric spaces $\mathrm{X}_{1}$ and $\mathrm{X}_{2}$ with respect to $\mathrm{G}$, let us denote by $\rho_{1}$ and $\rho_{2}$ the corresponding associated unitary representations. Then we assign to each $\Lambda$ in $\operatorname{Hom}\left(L_{2}\left(\mathrm{X}_{1}\right), L_{2}\left(\mathrm{X}_{2}\right)\right)$ its kernel $\lambda: \mathrm{X}_{2} \times \mathrm{X}_{1} \rightarrow \mathbb{C}$ with respect to the natural bases. The mapping $\Lambda \rightarrow \lambda$ is clearly a linear isomorphism $\Psi: \operatorname{Hom}\left(L_{2}\left(\mathrm{X}_{1}\right), L_{2}\left(\mathrm{X}_{2}\right)\right) \rightarrow L_{2}\left(\mathrm{X}_{2} \times \mathrm{X}_{1}\right)$ with $\Lambda$ and $\lambda$ related by

$$
\Lambda \varphi\left(x_{2}\right)=\sum_{x_{1} \in \mathrm{X}_{1}} \lambda\left(x_{2}, x_{1}\right) \varphi\left(x_{1}\right) .
$$

Let $\operatorname{Hom}_{\mathrm{G}}\left(L_{2}\left(\mathrm{X}_{1}\right), L_{2}\left(\mathrm{X}_{2}\right)\right)$ be the space of intertwining maps for $\rho_{1}$ and $\rho_{2}$. If we compare $\Lambda \circ \rho_{1}(g)$ and $\rho_{2}(g) \circ \Lambda$ written in terms of $\lambda$, it is not difficult to check that $\Lambda \in \operatorname{Hom}_{\mathrm{G}}\left(L_{2}\left(\mathrm{X}_{1}\right), L_{2}\left(\mathrm{X}_{2}\right)\right)$ if and only if $\lambda\left(g x_{2}, g x_{1}\right)=\lambda\left(x_{2}, x_{1}\right)$ holds for all $g \in \mathrm{G}$ and all $\left(x_{1}, x_{2}\right) \in \mathrm{X}_{1} \times \mathrm{X}_{2}$. That is, $\Lambda$ is an intertwining operator for $\rho_{1}$ and $\rho_{2}$ if and only if $\lambda$ is constant on the orbits of the action

$$
\mathrm{G} \times \mathrm{X}_{2} \times \mathrm{X}_{1} \ni\left(g,\left(x_{2}, x_{1}\right)\right) \longmapsto\left(g x_{2}, g x_{1}\right) \in \mathrm{X}_{2} \times \mathrm{X}_{1} .
$$

Now, assume we are given a transitive action $\mathrm{G} \times \mathrm{X} \rightarrow \mathrm{X}$ of a finite group $\mathrm{G}$ on a finite set $\mathrm{X}$ endowed with a distance $\partial$. We say that $\mathrm{X}$ is a two-point homogeneous space when for any two pairs $\left(x_{1}, x_{2}\right),\left(y_{1}, y_{2}\right) \in \mathrm{X} \times \mathrm{X}$ satisfying $\partial\left(x_{1}, x_{2}\right)=\partial\left(y_{1}, y_{2}\right)$, there exists $g \in \mathrm{G}$ such that $g x_{1}=y_{1}$ and $g x_{2}=y_{2}$.

Remark 1.2. An action $\mathrm{G} \times \mathrm{X} \rightarrow \mathrm{X}$ is called symmetric when for any $x_{1}, x_{2} \in \mathrm{X}$ there exists $g \in \mathrm{G}$ such that $g x_{1}=x_{2}$ and $g x_{2}=x_{1}$. Any two-point homogeneous space $\mathrm{X}$ is clearly equipped with a symmetric action and in that case $\mathrm{X}$ becomes a finite symmetric space with respect to G. Namely, if the action of $G$ on $\mathrm{X}$ is symmetric, then $\Psi\left(\operatorname{End}_{\mathrm{G}}\left(L_{2}(\mathrm{X})\right)\right)$ is a subalgebra of $L_{2}(\mathrm{X} \times \mathrm{X})$ made up of symmetric matrices, hence abelian. Finally, since the mapping $\Psi$ is an algebra isomorphism when $\mathrm{X}_{1}=\mathrm{X}_{2}$, it turns out that $\mathrm{X}$ is a finite symmetric space.

Let us write $\widehat{\mathrm{G}}$ for the dual object of $\mathrm{G}$. That is, the set of irreducible unitary representations of $\mathrm{G}$ up to unitary equivalence. Let us consider the set

$$
\widehat{\mathrm{G}}_{\mathrm{X}}=\left\{\pi \in \widehat{\mathrm{G}}: \operatorname{Mult}_{\pi}(\rho) \neq 0\right\} .
$$

Notice that if $\mathrm{X}$ is symmetric with respect to $\mathrm{G}$, then every $\pi \in \widehat{\mathrm{G}}_{\mathrm{X}}$ satisfies $\operatorname{Mult}_{\pi}(\rho)=1$ since $\rho$ is multiplicity-free by Remark 1.1 This set allows us to decompose $L_{2}(\mathrm{X})$ into irreducible components

$$
L_{2}(\mathrm{X})=\bigoplus_{\pi \in \widehat{\mathrm{G}}_{\mathrm{X}}} L_{2}(\mathrm{X})_{\pi}
$$

We denote by $\mathrm{P}_{\pi}$ the orthogonal projection onto $L_{2}(\mathrm{X})_{\pi}$. The kernel of $\mathrm{P}_{\pi}$ will be denoted by $p_{\pi}$. The spherical functions on $\mathrm{X}$ are defined by

$$
\psi_{\mathrm{X}, \pi}=\frac{|\mathrm{X}|}{d(\pi)} p_{\pi} \in \Psi\left(\operatorname{End}_{\mathrm{G}}\left(L_{2}(\mathrm{X})\right)\right)
$$


where $\pi \in \widehat{\mathrm{G}}_{\mathrm{X}}$ and $d(\pi)$ denotes the degree of $\pi$. We shall also write $\mathrm{S}_{\mathrm{X}, \pi}$ for the associated operator in $\operatorname{End}_{\mathrm{G}}\left(L_{2}(\mathrm{X})\right)$ with kernel $\psi_{\mathrm{X}, \pi}$. A slightly modified version of the following result can be found in Terra's book [11, Th. 1 of Chapter 20].

Theorem 1.3. Let $\mathrm{X}$ be a finite symmetric space with respect to the finite group $\mathrm{G}$ and let $\psi \in \Psi\left(\operatorname{End}_{\mathrm{G}}\left(L_{2}(\mathrm{X})\right)\right)$, then the following are equivalent:

(a) There exists $\pi \in \widehat{\mathrm{G}}_{\mathrm{X}}$ such that $\psi=\psi_{\mathrm{X}, \pi}$.

(b) The function $\psi$ satisfies $\psi\left(x_{0}, x_{0}\right)=1$ for all $x_{0} \in \mathrm{X}$ and

$$
\frac{1}{\left|\mathrm{G}_{x_{0}}\right|} \sum_{g \in \mathrm{G}_{x_{0}}} \psi\left(g x_{1}, x_{2}\right)=\psi\left(x_{1}, x_{0}\right) \psi\left(x_{0}, x_{2}\right)=\frac{1}{\left|\mathrm{G}_{x_{0}}\right|} \sum_{g \in \mathrm{G}_{x_{0}}} \psi\left(x_{1}, g x_{2}\right)
$$

for every $x_{1}, x_{2} \in \mathrm{X}$ and where $\mathrm{G}_{x_{0}}$ denotes the isotropy subgroup of $x_{0}$.

1.2. Notation and results from $q$-combinatorics. We shall also need some results from $q$-combinatorics that we summarize here. Our notation will follow the book [6] by Gasper and Rahman. For some related results in $q$-combinatorics, the reader is referred to [7]. The $q$-shifted factorials are defined as follows

$$
(u ; q)_{k}=\prod_{j=0}^{k-1}\left(1-q^{j} u\right) \quad \text { and } \quad\left(u ; q^{-1}\right)_{k}=\prod_{j=0}^{k-1}\left(1-q^{-j} u\right) .
$$

Then, the $q$-binomial coefficients can be written as

$$
\left[\begin{array}{c}
m \\
k
\end{array}\right]_{q}=\frac{(q ; q)_{m}}{(q ; q)_{k}(q ; q)_{m-k}}=\frac{\left(q^{m} ; q^{-1}\right)_{k}}{\left(q^{k} ; q^{-1}\right)_{k}}=\left[\begin{array}{c}
m \\
m-k
\end{array}\right]_{q} .
$$

Now we present some well-known combinatorial identities that will be used in this paper with no further comment. Let us recall some of the objects introduced above: $\mathbb{K}, \Omega, \mathrm{X}_{r}, \mathrm{~V}_{r}, \partial, \ldots$ Besides, let us consider the general linear group $\operatorname{GL}(n, \mathbb{K})$. That is, the group of endomorphisms of $\Omega$. Then, we have

$$
\begin{aligned}
|\mathrm{GL}(n, \mathbb{K})| & =(-1)^{n} q^{\left(\begin{array}{c}
n \\
2
\end{array}\right)(q ; q)_{n},} \\
\operatorname{dim} \mathrm{V}_{r} & =\left[\begin{array}{l}
n \\
r
\end{array}\right]_{q} .
\end{aligned}
$$

Moreover, given $0 \leq r_{1} \leq r \leq r_{2} \leq n$ and $\left(x_{1}, x_{2}\right) \in \mathrm{X}_{r_{1}} \times \mathrm{X}_{r_{2}}$, we have

$$
\left|\left\{x \in \mathrm{X}_{r} \mid x_{1} \subset x \subset x_{2}\right\}\right|=\left[\begin{array}{c}
r_{2}-r_{1} \\
r-r_{1}
\end{array}\right]_{q} .
$$

Eventually, we shall also use the $q$-multinomial coefficients

$$
\left[\begin{array}{c}
m \\
r_{1}, r_{2}, \ldots, r_{k}
\end{array}\right]_{q}=\frac{(q ; q)_{m}}{(q ; q)_{r_{1}} \cdots(q ; q)_{r_{k}}}
$$

with $m=\sum_{1}^{k} r_{j}$ and $r_{1}, r_{2}, \ldots, r_{k} \geq 0$. The combinatorial interpretation is

$$
\left|\left\{\left(x_{1}, \ldots, x_{k}\right) \in \prod_{j=1}^{k} \mathrm{X}_{r_{j}} \mid \Omega=\bigoplus_{j=1}^{k} x_{j}\right\}\right|=\left[\begin{array}{c}
n \\
r_{1}, \ldots, r_{k}
\end{array}\right]_{q} \prod_{i<j} q^{r_{i} r_{j}} .
$$

In particular, given $z \in \mathrm{X}_{r}$ we have

$$
\left|\left\{w \in \mathrm{X}_{n-r} \mid \Omega=z \oplus w\right\}\right|=q^{r(n-r)} .
$$


Let $\mathrm{I}_{n}\left(r_{1}, r_{2}\right)$ be the set of all possible values of the parameter $\partial\left(x_{2}, x_{1}\right)$ for $x_{1} \in \mathrm{X}_{r_{1}}$ and $x_{2} \in \mathrm{X}_{r_{2}}$. Given $t \in \mathrm{I}_{n}\left(r_{1}, r_{2}\right)$, the following identity follows from the relations above and will be very useful for our forthcoming computations

$$
\begin{aligned}
& \left|\left\{\left(x_{1}, x_{2}\right) \in \mathrm{X}_{r_{1}} \times \mathrm{X}_{r_{2}} \mid \partial\left(x_{2}, x_{1}\right)=t\right\}\right| \\
& \quad=q^{t\left(r_{1}-r_{2}+t\right)}\left[t, r_{2}-t, r_{1}-r_{2}+t, n-r_{1}-t\right]_{q} .
\end{aligned}
$$

Remark 1.4. We shall also use the notation $\left(u_{1}, u_{2}, \ldots, u_{d} ; q\right)_{k}=\prod_{j=1}^{d}\left(u_{j} ; q\right)_{k}$.

1.3. The basis of the algebra $\operatorname{End}_{G}(V)$. Let $G$ be the general linear group $\mathrm{GL}(n, \mathbb{K})$ of endomorphisms of $\Omega$ considered above. This group acts naturally on the set $\mathrm{X}$ of linear subspaces of the vector space $\Omega$. The orbits of this action are the subsets $\mathrm{X}_{0}, \mathrm{X}_{1}, \ldots, \mathrm{X}_{n}$ of linear subspaces of dimensions $0,1, \ldots, n$.

Remark 1.5. The action of $\mathrm{G}$ on $\mathrm{X}_{r}$ preserves $\partial_{r}$ and $\mathrm{X}_{r}$ is clearly a two-point homogeneous space for each $0 \leq r \leq n$. In particular, by Remark $1.2 \mathrm{X}_{r}$ becomes a finite symmetric space with respect to G. Besides, Remark 1.1 gives that the left regular representations of $\mathrm{G}$ into $\mathrm{V}_{r}$ are multiplicity-free for each $0 \leq r \leq n$.

Given any two integers $0 \leq r_{1}, r_{2} \leq n$, we shall identify each space $\operatorname{Hom}\left(\mathrm{V}_{r_{1}}, \mathrm{~V}_{r_{2}}\right)$ with a subspace of End(V) by right multiplication by the orthogonal projection from $\mathrm{V}$ onto $\mathrm{V}_{r_{1}}$. Applying the same identification for the intertwining operators, we obtain the following decompositions

$$
\begin{aligned}
\operatorname{End}(\mathrm{V}) & =\bigoplus_{r_{1}, r_{2}} \operatorname{Hom}\left(\mathrm{V}_{r_{1}}, \mathrm{~V}_{r_{2}}\right) \\
\operatorname{End}_{\mathrm{G}}(\mathrm{V}) & =\bigoplus_{r_{1}, r_{2}} \operatorname{Hom}_{\mathrm{G}}\left(\mathrm{V}_{r_{1}}, \mathrm{~V}_{r_{2}}\right) .
\end{aligned}
$$

The algebra End(V) is a Hilbert space with respect to the Hilbert-Schmidt inner product and the direct sums given above become orthogonal decompositions with respect to this structure. Besides, we know that the kernel of any intertwining operator $\Lambda \in \operatorname{Hom}_{\mathrm{G}}\left(\mathrm{V}_{r_{1}}, \mathrm{~V}_{r_{2}}\right)$ is constant on the orbits of $\left(g,\left(x_{2}, x_{1}\right)\right) \mapsto\left(g x_{2}, g x_{1}\right)$. These orbits are completely determined by the parameter $\partial\left(x_{2}, x_{1}\right)$. In particular, the kernel of $\Lambda$ can be regarded as a function $\lambda: \mathrm{I}_{n}\left(r_{1}, r_{2}\right) \rightarrow \mathbb{C}$, where the index set $\mathrm{I}_{n}\left(r_{1}, r_{2}\right)$ was considered above

$\mathrm{I}_{n}\left(r_{1}, r_{2}\right)=\left\{\partial\left(x_{2}, x_{1}\right) \mid x_{1} \in \mathrm{X}_{r_{1}}, x_{2} \in \mathrm{X}_{r_{2}}\right\}=\left\{0 \vee\left(r_{2}-r_{1}\right) \leq t \leq r_{2} \wedge\left(n-r_{1}\right)\right\}$.

Here $\wedge$ stands for min and $\vee$ for max. Therefore we have

$$
\Lambda \varphi\left(x_{2}\right)=\sum_{x_{1} \in \mathrm{X}_{r_{1}}} \lambda\left(\partial\left(x_{2}, x_{1}\right)\right) \varphi\left(x_{1}\right)
$$

for any $\varphi \in \mathrm{V}_{r_{1}}$ and

$$
\operatorname{dim}\left(\operatorname{Hom}_{\mathrm{G}}\left(\mathrm{V}_{r_{1}}, \mathrm{~V}_{r_{2}}\right)\right)=\left|\mathrm{I}_{n}\left(r_{1}, r_{2}\right)\right|=1+\mathrm{N}\left(r_{1}, r_{2}\right),
$$

where $\mathrm{N}\left(r_{1}, r_{2}\right)=r_{1} \wedge r_{2} \wedge\left(n-r_{1}\right) \wedge\left(n-r_{2}\right)$. Reciprocally, any $\lambda: \mathrm{I}_{n}\left(r_{1}, r_{2}\right) \rightarrow \mathbb{C}$ determines an operator $\Lambda \in \operatorname{Hom}_{\mathrm{G}}\left(\mathrm{V}_{r_{1}}, \mathrm{~V}_{r_{2}}\right)$. Moreover, since $\mathrm{V}_{r}$ is multiplicity free, Schur lemma gives that the dimension of $\operatorname{Hom}_{\mathrm{G}}\left(\mathrm{V}_{r_{1}}, \mathrm{~V}_{r_{2}}\right)$ is the number of irreducible components that $\mathrm{V}_{r_{1}}$ and $\mathrm{V}_{r_{2}}$ have in common. In particular, we have 
- $\mathrm{V}_{r}$ has $1+\mathrm{N}(r, r)=1+r \wedge(n-r)$ irreducible components.

- The number of irreducible components that $\mathrm{V}_{r_{1}}$ and $\mathrm{V}_{r_{2}}$ have in common is the minimum of the numbers of irreducible components of $\mathrm{V}_{r_{1}}$ and $\mathrm{V}_{r_{2}}$.

Therefore, there exist a family of inequivalent irreducible unitary representations $\pi_{s}: \mathrm{G} \rightarrow \mathcal{B}\left(\mathcal{H}_{s}\right)$ such that, if we denote by $\mathrm{V}_{r, s}$ the G-invariant subspace of $\mathrm{V}_{r}$ equivalent to $\mathcal{H}_{s}$, the left regular representation $\rho_{r}: \mathrm{G} \rightarrow \mathcal{B}\left(\mathrm{V}_{r}\right)$ and the Hilbert space $\mathrm{V}_{r}$ decompose into irreducibles as follows

$$
\rho_{r} \simeq \bigoplus_{s=0}^{r \wedge(n-r)} \pi_{s} \quad \text { and } \quad \mathrm{V}_{r}=\bigoplus_{s=0}^{r \wedge(n-r)} \mathrm{V}_{r, s}
$$

Moreover, the representations of $\mathrm{G}$ into $\mathrm{V}_{r_{1}, s_{1}}$ and $\mathrm{V}_{r_{2}, s_{2}}$ are equivalent if and only if $s_{1}=s_{2}$. Finally we note that

$$
\operatorname{dim} \mathrm{V}_{r, s}=\operatorname{dim} \mathrm{V}_{s, s}=\operatorname{dim} \mathrm{V}_{s}-\operatorname{dim} \mathrm{V}_{s-1}=\left[\begin{array}{l}
n \\
s
\end{array}\right]_{q}-\left[\begin{array}{c}
n \\
s-1
\end{array}\right]_{q} .
$$

Here we assume by convention $\mathrm{X}_{-1}=\emptyset$, so that $\operatorname{dim} \mathrm{V}_{-1}=0$. The last identity in (66) follows from relation (21). By Schur lemma we know that $\operatorname{Hom}_{\mathrm{G}}\left(\mathrm{V}_{r_{1}, s}, \mathrm{~V}_{r_{2}, s}\right)$ is one-dimensional. In summary, we have obtained an orthogonal decomposition of the algebra of intertwining operators $\operatorname{End}_{G}(V)$ into one-dimensional subspaces

$$
\operatorname{End}_{\mathrm{G}}(\mathrm{V})=\bigoplus_{0 \leq r_{1}, r_{2} \leq n} \bigoplus_{s=0}^{\mathrm{N}\left(r_{1}, r_{2}\right)} \operatorname{Hom}_{\mathrm{G}}\left(\mathrm{V}_{r_{1}, s}, \mathrm{~V}_{r_{2}, s}\right)
$$

This decomposition provides a natural basis of the algebra $\operatorname{End}_{G}(V)$ which will be the object of our study. Namely, taking a non-zero element $\Lambda_{s}^{r_{1}, r_{2}}$ in each space $\operatorname{Hom}_{\mathrm{G}}\left(\mathrm{V}_{r_{1}, s}, \mathrm{~V}_{r_{2}, s}\right)$, we obtain a basis $\mathbf{B}$ of the algebra $\operatorname{End}_{\mathrm{G}}(\mathrm{V})$. Our definition of $\Lambda_{s}^{r_{1}, r_{2}}$ is still ambiguous since we have only defined it up to a constant factor. We shall precise this below. As it was announced in the Introduction, the aim of this paper is to provide several expressions for the kernels $\lambda_{s}^{r_{1}, r_{2}}$ and to give an explicit formula for the mapping product $\Lambda_{s}^{r_{2}, r_{3}} \circ \Lambda_{s}^{r_{1}, r_{2}}$.

\section{LAPLACIAN OPERATORS ON GRAPHS}

In this section we deal with some Laplacian type operators which will be useful to identify certain difference equation satisfied by the kernels of $\Lambda_{s}^{r_{1}, r_{2}}$ in the usual rank of parameters for $r_{1}, r_{2}$ and $s$. We begin by recalling some general results for Laplacian operators on graphs. Then we focus on the Grassmann graphs.

2.1. General results. Let $X$ be a finite distance-regular graph and let $\partial$ be the distance on X. Assume there exists a finite group G acting on X such that the graph $\mathrm{X}$ becomes a two-point homogeneous space with respect to $\mathrm{G}$. This structure on $\mathrm{X}$ allows us to define two Laplacian type operators on the vector space $\mathrm{V}$ of complex valued functions $\varphi: \mathrm{X} \rightarrow \mathbb{C}$. First, given $x \in \mathrm{X}$, we consider the set

$$
\mathbf{S}_{1}(x)=\{y \in \mathrm{X} \mid \partial(x, y)=1\} \text {. }
$$

The regularity for the distance imposed on the graph $\mathrm{X}$ implies that the cardinality of the set $\mathbf{S}_{1}(x)$ does not depend on $x$. This cardinality $\operatorname{val}(\mathrm{X})$ is called in the 
literature the valence of $\mathrm{X}$. The graph Laplacian $\mathcal{L}_{\mathrm{X}}: \mathrm{V} \rightarrow \mathrm{V}$ is the operator defined as follows

$$
\mathcal{L}_{\mathrm{X}} \varphi(x)=\sum_{y \in \mathbf{S}_{1}(x)}(\varphi(y)-\varphi(x))=\sum_{y \in \mathbf{S}_{1}(x)} \varphi(y)-\operatorname{val}(\mathrm{X}) \varphi(x) .
$$

Second, let us consider a subset $\mathbf{T}$ of $\mathrm{G}$ satisfying the following properties

- $\mathbf{T}=\mathbf{T}^{-1}$.

- $\mathbf{T}=g \mathbf{T} g^{-1}$ for all $g \in \mathrm{G}$.

- $\partial(x, h x) \leq 1$ for all $x \in \mathrm{X}$ and all $h \in \mathbf{T}$.

- There exists $x \in \mathrm{X}$ such that $\mathbf{T} \nsubseteq \mathrm{G}_{x}$, the isotropy subgroup of $x$.

The group Laplacian $\mathcal{L}_{\mathbf{T}, \mathrm{X}}: \mathrm{V} \rightarrow \mathrm{V}$ is defined as follows

$$
\mathcal{L}_{\mathbf{T}, \mathrm{X}} \varphi(x)=\sum_{h \in \mathbf{T}}(\varphi(h x)-\varphi(x))=\sum_{h \in \mathbf{T}} \varphi(h x)-|\mathbf{T}| \varphi(x) .
$$

Both the graph and the group Laplacians are self-adjoint operators with respect to the natural inner product on $\mathrm{V}$. This is an easy exercise that we leave to the reader. Besides, recalling that an endomorphism of $\mathrm{V}$ is an intertwining operator if and only if its kernel is constant on the orbits of the action of $\mathrm{G}$ on $\mathrm{X} \times \mathrm{X}$, it is not difficult to check that both the graph and the group Laplacians belong to the intertwining algebra $\operatorname{End}_{\mathrm{G}}(\mathrm{V})$. Now let $\mathbf{T}$ be a subset of $\mathrm{G}$ satisfying the properties above. Then, given $x \in \mathrm{X}$ and $y \in \mathbf{S}_{1}(x)$, we define

$$
\begin{aligned}
\mathbf{T}_{x} & =\{h \in \mathbf{T} \mid h x=x\}, \\
\mathbf{T}_{x, y} & =\{h \in \mathbf{T} \mid h x=y\} .
\end{aligned}
$$

From the properties of $\mathbf{T}$ it follows that $g \mathbf{T}_{x} g^{-1}=\mathbf{T}_{g x}$ and $g \mathbf{T}_{x, y} g^{-1}=\mathbf{T}_{g x, g y}$ for all $g \in \mathrm{G}$. In particular, since the distance-regular graph $\mathrm{X}$ is assumed to be a two-point homogeneous space, the numbers

$$
\begin{aligned}
\gamma_{0}(\mathbf{T}, \mathrm{X}) & =\left|\mathbf{T}_{x}\right|, \\
\gamma_{1}(\mathbf{T}, \mathrm{X}) & =\left|\mathbf{T}_{x, y}\right|,
\end{aligned}
$$

do not depend on the election of $x \in \mathrm{X}$ and $y \in \mathbf{S}_{1}(x)$. We now state some basic results on these Laplacian operators that will be used in the sequel. Notice that the fourth condition imposed on $\mathbf{T}$ implies that $\gamma_{1}(\mathbf{T}, \mathbf{X})>0$.

Lemma 2.1. $|\mathbf{T}|=\gamma_{0}(\mathbf{T}, \mathrm{X})+\operatorname{val}(\mathrm{X}) \gamma_{1}(\mathbf{T}, \mathrm{X})$ and $\mathcal{L}_{\mathbf{T}, \mathrm{X}}=\gamma_{1}(\mathbf{T}, \mathrm{X}) \mathcal{L}_{\mathrm{X}}$.

Proof. Obviously we have

$$
\mathbf{T}=\mathbf{T}_{x} \cup\left[\bigcup_{y \in \mathbf{S}_{1}(x)} \mathbf{T}_{x, y}\right]
$$

with disjoint unions. Hence, the first assertion follows. Besides, we notice that

$$
\mathcal{L}_{\mathbf{T}, \mathrm{X}} \varphi(x)=\sum_{h \in \mathbf{T}}(\varphi(h x)-\varphi(x))=\sum_{y \in \mathbf{S}_{1}(x)} \sum_{h \in \mathbf{T}_{x, y}}(\varphi(h x)-\varphi(x)) .
$$

By definition, the last expression is $\gamma_{1}(\mathbf{T}, \mathrm{X}) \mathcal{L}_{\mathrm{X}} \varphi(x)$. This concludes the proof. 
Lemma 2.2. Let $\mathrm{W}$ be a $\mathrm{G}$-invariant subspace of $\mathrm{V}$. Then, the group Laplacian $\mathcal{L}_{\mathbf{T}, \mathrm{X}}$ preserves $\mathrm{W}$. Besides, if $\mathrm{W}$ is irreducible, there exists a complex number $\mu$ depending only on the representation of $\mathrm{G}$ into $\mathrm{W}$ such that

$$
\mathcal{L}_{\mathbf{T}, \mathrm{X}_{\mid \mathrm{W}}}+\mu 1_{\mathrm{W}}=0 .
$$

Proof. Let $\rho: \mathrm{G} \rightarrow \mathcal{B}(\mathrm{V})$ be the left regular representation associated to the action of $\mathrm{G}$ into $\mathrm{X}$. Then, for any $\varphi \in \mathrm{W}$, we can write

$$
\mathcal{L}_{\mathbf{T}, \mathbf{X}} \varphi(x)=\sum_{h \in \mathbf{T}}\left(\rho(h) \varphi(x)-1_{\mathrm{V}} \varphi(x)\right) .
$$

The first claim follows from the relation above. The second is a consequence of Schur lemma. Namely, if $\mathrm{W}$ is irreducible and $\pi: \mathrm{G} \rightarrow \mathcal{B}(\mathrm{W})$ denotes the restriction of $\rho$ to $\mathrm{W}$, we have

$$
\mathcal{L}_{\mathbf{T}, \mathrm{X}_{\mid \mathrm{W}}}=\sum_{h \in \mathbf{T}}\left(\pi(h)-1_{\mathrm{W}}\right) .
$$

Therefore, since this is an intertwining operator with respect to $\pi$, Schur lemma gives that $\mathcal{L}_{\mathbf{T}, \mathrm{X} \mid \mathrm{W}}+\mu 1_{\mathrm{W}}=0$ with $\mu$ depending only on the representation $\pi$.

2.2. Laplacian operators on the graphs $\mathrm{X}_{r}$. In this paragraph we return to the study of the intertwining algebra $\operatorname{End}_{G}(V)$ described in Section 1 In particular, we shall work with the general linear group $\mathrm{G}$ of endomorphisms of $\Omega$ and the Grassmann graphs $\mathrm{X}_{r}$ for $0 \leq r \leq n$. We denote by $\mathcal{L}_{r}: \mathrm{V}_{r} \rightarrow \mathrm{V}_{r}$ the graph Laplacian on $\mathrm{X}_{r}$ while $\mathcal{L}_{\mathbf{T}, r}: \mathrm{V}_{r} \rightarrow \mathrm{V}_{r}$ stands for the group Laplacian. If $\operatorname{rk}(\Lambda)$ denotes the rank of a mapping $\Lambda$, we consider the subset $\mathbf{T}$ of $\mathrm{G}$ defined as follows

$$
\begin{aligned}
\mathbf{T} & =\left\{g \in \mathrm{G} \mid \operatorname{rk}(g-1)=1,(g-1)^{2}=0\right\} \\
& =\left\{1+\omega \otimes \alpha \mid \alpha \in \Omega^{*} \backslash\{0\}, \omega \in \operatorname{Ker}(\alpha) \backslash\{0\}\right\},
\end{aligned}
$$

where $\omega \otimes \alpha \in \operatorname{End}(\Omega)$ is given by $(\omega \otimes \alpha)\left(\omega_{0}\right)=\alpha\left(\omega_{0}\right) \omega$. We need to check that the subset $\mathbf{T}$ satisfies the properties introduced before the definition of the group Laplacian. But this is an easy exercise that we leave to the reader. The main results of this section are summarized in the following theorem. We shall also need to consider the operator $\mathcal{L}_{r_{1}, r_{2}}: \operatorname{Hom}_{\mathrm{G}}\left(\mathrm{V}_{r_{1}}, \mathrm{~V}_{r_{2}}\right) \rightarrow \operatorname{Hom}_{\mathrm{G}}\left(\mathrm{V}_{r_{1}}, \mathrm{~V}_{r_{2}}\right)$ defined by the following relation

$$
\mathcal{L}_{r_{1}, r_{2}} \Lambda=\mathcal{L}_{r_{2}} \circ \Lambda .
$$

Theorem 2.3. The Laplacian operators considered above satisfy:

(a) We have

$$
\gamma_{1}\left(\mathbf{T}, \mathrm{X}_{r}\right)=q^{n-2}(q-1) \quad \text { and } \quad \mathcal{L}_{\mathbf{T}, r}=q^{n-2}(q-1) \mathcal{L}_{r} .
$$

(b) The mappings $\Lambda \in \operatorname{Hom}_{\mathrm{G}}\left(\mathrm{V}_{r_{1}}, \mathrm{~V}_{r_{2}}\right)$ satisfy

$$
\mathcal{L}_{r_{2}} \circ \Lambda=\mathcal{L}_{r_{1}, r_{2}} \Lambda=\Lambda \circ \mathcal{L}_{r_{1}} .
$$

(c) Given $\varphi \in \mathrm{V}_{r, s}$ and $\Lambda \in \operatorname{Hom}_{\mathrm{G}}\left(\mathrm{V}_{r_{1}, s}, \mathrm{~V}_{r_{2}, s}\right)$, there exists $\mu_{s}(n)$ such that

$$
\begin{aligned}
\mathcal{L}_{r} \varphi(x)+\mu_{s}(n) \varphi(x) & =0 \\
\mathcal{L}_{r_{1}, r_{2}} \Lambda+\mu_{s}(n) \Lambda & =0 .
\end{aligned}
$$


(d) If $\Lambda \in \operatorname{Hom}_{\mathrm{G}}\left(\mathrm{V}_{r_{1}}, \mathrm{~V}_{r_{2}}\right)$ has kernel $\lambda: \mathrm{I}_{n}\left(r_{1}, r_{2}\right) \rightarrow \mathbb{C}$, then

$$
\Lambda^{\prime}=\mathcal{L}_{r_{1}, r_{2}} \Lambda
$$

has kernel $\lambda^{\prime}: \mathrm{I}_{n}\left(r_{1}, r_{2}\right) \rightarrow \mathbb{C}$ given by

$$
\lambda^{\prime}(t)=\mathbf{b}_{r_{1}, r_{2}}(t)(\lambda(t+1)-\lambda(t))+\mathbf{c}_{r_{1}, r_{2}}(t)(\lambda(t-1)-\lambda(t)),
$$

where, given $\left(x_{1}, x_{2}\right) \in \mathrm{X}_{r_{1}} \times \mathrm{X}_{r_{2}}$ with $\partial\left(x_{2}, x_{1}\right)=t$, we have

$$
\begin{aligned}
& \mathbf{b}_{r_{1}, r_{2}}(t)=\left|\left\{y \in \mathrm{X}_{r_{2}} \mid \partial\left(y, x_{2}\right)=1, \partial\left(y, x_{1}\right)=t+1\right\}\right|, \\
& \mathbf{c}_{r_{1}, r_{2}}(t)=\left|\left\{y \in \mathrm{X}_{r_{2}} \mid \partial\left(y, x_{2}\right)=1, \partial\left(y, x_{1}\right)=t-1\right\}\right| .
\end{aligned}
$$

(e) The following expressions hold

$$
\begin{aligned}
& \mathbf{b}_{r_{1}, r_{2}}(t)=\frac{q^{r_{1}-r_{2}+1}\left(q^{t}-q^{r_{2}}\right)\left(q^{t}-q^{n-r_{1}}\right)}{(q-1)^{2}}, \\
& \mathbf{c}_{r_{1}, r_{2}}(t)=\frac{q^{r_{1}-r_{2}}\left(q^{t}-1\right)\left(q^{t}-q^{r_{2}-r_{1}}\right)}{(q-1)^{2}} .
\end{aligned}
$$

The proof of Theorem 2.3 requires several auxiliary results. We shall state and prove these results as they are needed. Given $x \in \mathrm{X}_{r}$, we consider the sets

$$
\begin{aligned}
& \mathbf{S}_{1}(x)=\left\{y \in \mathbf{X}_{r} \mid \partial_{r}(x, y)=1\right\} \\
& \widetilde{\mathbf{S}}_{1}(x)=\left\{(z, \omega) \in \mathrm{X}_{r-1} \times \Omega \mid z \subset x, \omega \notin x\right\} .
\end{aligned}
$$

Lemma 2.4. Given any $x \in \mathrm{X}_{r}$, the mapping

$$
(z, \omega) \in \widetilde{\mathbf{S}}_{1}(x) \longmapsto y=z \oplus \mathbb{K} \omega \in \mathbf{S}_{1}(x)
$$

is surjective, $z=x \cap y$ and there exist $q^{r-1}(q-1)$ possible $\omega$ for each $y \in \mathbf{S}_{1}(x)$.

Proof. Given $x, y \in \mathrm{X}_{r}$ we have $\partial_{r}(x, y)=r-\partial(x \cap y)$. Hence $\partial_{r}(x, y)=1$ is equivalent to $\partial(x \cap y)=r-1$. In other words, $y=z \oplus \mathbb{K} \omega$ with

$$
z=x \cap y \quad \text { and } \quad \omega \in y \backslash z \text {. }
$$

Hence we have $\omega \notin x,(z, \omega) \in \widetilde{\mathbf{S}}_{1}(x)$ and there exists

$$
|y \backslash z|=q^{r}-q^{r-1}
$$

possible elections for the vector $\omega$. Therefore, the proof is completed.

Lemma 2.4 allows us to compute the valence of the Grassmann graphs $\mathrm{X}_{r}$. Namely, since $\left|\mathrm{X}_{r}\right|=\operatorname{dim} \mathrm{V}_{r}$, we can apply (2) to obtain for $x \in \mathrm{X}_{r}$

$$
\operatorname{val}\left(\mathbf{X}_{r}\right)=\left|\mathbf{S}_{1}(x)\right|=\frac{\left|\widetilde{\mathbf{S}}_{1}(x)\right|}{q^{r-1}(q-1)}=\left[\begin{array}{l}
r \\
1
\end{array}\right]_{q} \frac{q^{n}-q^{r}}{q^{r-1}(q-1)}=\frac{q\left(q^{r}-1\right)\left(q^{n-r}-1\right)}{(q-1)^{2}}
$$

Lemma 2.5. Given $g=1+\omega \otimes \alpha \in \mathbf{T}$, we have $g x=x$ iff $x \subset \operatorname{Ker}(\alpha)$ or $\omega \in x$.

Proof. If $x \subset \operatorname{Ker}(\alpha)$, it is clear that $g x=x$. Besides, if $x$ is not a subspace of $\operatorname{Ker}(\alpha)$ but $\omega \in x$, we have $x=x^{\prime} \oplus \mathbb{K} \omega^{\prime}$ with $x^{\prime}=x \cap \operatorname{Ker}(\alpha)$ and $\omega^{\prime} \in x \backslash \operatorname{Ker}(\alpha)$. This gives

$$
g x=x^{\prime} \oplus \mathbb{K} g\left(\omega^{\prime}\right)=x^{\prime} \oplus \mathbb{K}\left(\omega^{\prime}+\alpha\left(\omega^{\prime}\right) \omega\right)=x^{\prime} \oplus \mathbb{K} \omega^{\prime}=x .
$$

Reciprocally, if $g x=x$ and $x$ does not belong to $\operatorname{Ker}(\alpha)$, we take $\omega^{\prime \prime} \in x \backslash \operatorname{Ker}(\alpha)$ so that $g\left(\omega^{\prime \prime}\right)=\omega^{\prime \prime}+\alpha\left(\omega^{\prime \prime}\right) \omega \in x$. Therefore, we conclude that $\omega \in x$. 
Now we combine the expression for the valence of $\mathrm{X}_{r}$ given in (7) with (2) and Lemma 2.5 to obtain the value of $\gamma_{1}\left(\mathbf{T}, \mathrm{X}_{r}\right)$

$$
\begin{aligned}
\operatorname{val}\left(\mathrm{X}_{r}\right) \gamma_{1}\left(\mathbf{T}, \mathrm{X}_{r}\right) & =|\{g \in \mathbf{T} \mid g x \neq x\}| \\
& =\left|\left\{1+\omega \otimes \alpha \mid \alpha \in \Omega^{*} \backslash\{0\}, \omega \in \operatorname{Ker}(\alpha) \backslash x, x \nsubseteq \operatorname{Ker}(\alpha)\right\}\right| \\
& =\left(\left[\begin{array}{c}
n \\
1
\end{array}\right]_{q}-\left[\begin{array}{c}
n-r \\
1
\end{array}\right]_{q}\right)\left(q^{n-1}-q^{r-1}\right) .
\end{aligned}
$$

Dividing on the right hand side by the value for $\operatorname{val}\left(\mathrm{X}_{r}\right)$ given in (7), we obtain the identity $\gamma_{1}\left(\mathbf{T}, \mathrm{X}_{r}\right)=q^{n-2}(q-1)$. This proves the first assertion of (a) in Theorem 2.3 The second assertion follows from Lemma 2.1. On the other hand, since any $\Lambda \in \operatorname{Hom}_{\mathrm{G}}\left(\mathrm{V}_{r_{1}}, \mathrm{~V}_{r_{2}}\right)$ commutes with the action of $\mathrm{G}$, we have

$$
\mathcal{L}_{\mathbf{T}, r_{2}} \circ \Lambda=\Lambda \circ \mathcal{L}_{\mathbf{T}, r_{1}} .
$$

Therefore, (b) in Theorem 2.3 follows from (a). Moreover, (c) is a consequence of Lemma 2.2 and (a). To prove (d), we take $\left(x_{1}, x_{2}\right) \in \mathrm{X}_{r_{1}} \times \mathrm{X}_{r_{2}}$ with $\partial\left(x_{2}, x_{1}\right)=t$. Then we have

$$
\begin{aligned}
\Lambda^{\prime} \varphi\left(x_{2}\right) & =\sum_{y \in \mathbf{S}_{1}\left(x_{2}\right)}\left(\Lambda \varphi(y)-\Lambda \varphi\left(x_{2}\right)\right) \\
& =\sum_{y \in \mathbf{S}_{1}\left(x_{2}\right)} \sum_{x_{1} \in \mathrm{X}_{r_{1}}}\left(\lambda\left(\partial\left(y, x_{1}\right)\right)-\lambda\left(\partial\left(x_{2}, x_{1}\right)\right)\right) \varphi\left(x_{1}\right) .
\end{aligned}
$$

In particular, we can write

$$
\lambda^{\prime}\left(\partial\left(x_{2}, x_{1}\right)\right)=\sum_{y \in \mathbf{S}_{1}\left(x_{2}\right)}\left(\lambda\left(\partial\left(y, x_{1}\right)\right)-\lambda\left(\partial\left(x_{2}, x_{1}\right)\right)\right) .
$$

Then (d) follows immediately from this. Finally, it remains to see (e). The proof requires two combinatorial lemmas. Let us notice that, given $\left(x_{1}, x_{2}\right) \in \mathrm{X}_{r_{1}} \times \mathrm{X}_{r_{2}}$ and $y \in \mathbf{S}_{1}\left(x_{2}\right)$, we have

$$
\begin{aligned}
\partial\left(x_{2}, x_{1}\right)-\partial\left(y, x_{1}\right) & =\partial\left(\left(x_{1}+x_{2}\right) / x_{1}\right)-\partial\left(\left(x_{1}+y\right) / x_{1}\right) \\
& =\partial\left(\left(x_{1}+x_{2}\right) /\left(x_{1}+z\right)\right)-\partial\left(\left(x_{1}+y\right) /\left(x_{1}+z\right)\right),
\end{aligned}
$$

with $z=x_{2} \cap y$. Besides, recalling that $y \in \mathbf{S}_{1}\left(x_{2}\right)$, it is not difficult to check that both dimensions appearing on the right hand side of (8) are either 0 or 1 . This remark will be used in the following results.

Lemma 2.6. Given $\left(x_{1}, x_{2}\right) \in \mathrm{X}_{r_{1}} \times \mathrm{X}_{r_{2}},(z, \omega) \in \widetilde{\mathbf{S}}_{1}\left(x_{2}\right)$ and $y=z \oplus \mathbb{K} \omega \in \mathbf{S}_{1}\left(x_{2}\right)$, the following assertions are equivalent:

(a) $\partial\left(y, x_{1}\right)=\partial\left(x_{2}, x_{1}\right)+1$.

(b) $x_{1}+z=x_{1}+x_{2}$ and $\omega \notin x_{1}+x_{2}$.

(c) $x_{1} \cap x_{2} \not \subset z$ and $\omega \notin x_{1}+x_{2}$.

Proof. Following (8) and the remark after it, we deduce (a) is equivalent to (b). On the other hand, since

$$
\partial\left(\left(x_{1}+x_{2}\right) /\left(x_{1}+z\right)\right)=1-\partial\left(x_{1} \cap x_{2}\right)+\partial\left(x_{1} \cap z\right),
$$

it follows that (b) is equivalent to (c). Therefore, the proof is completed. 
Notice that $z$ is a $\left(r_{2}-1\right)$-dimensional subspace of $x_{2}$. Besides, given $x_{2} \in \mathrm{X}_{r_{2}}$, the quotient mapping $\pi: x_{2} \rightarrow x_{2} /\left(x_{1} \cap x_{2}\right)$ provides the following identity

$\left|\left\{z \subset x_{2} \mid \partial(z)=r_{2}-1, x_{1} \cap x_{2} \subset z\right\}\right|=\left|\left\{z^{\prime} \subset x_{2} /\left(x_{1} \cap x_{2}\right) \mid \partial\left(z^{\prime}\right)=\partial\left(x_{2}, x_{1}\right)-1\right\}\right|$.

In other words, the number of subspaces $z$ of $x_{2}$ satisfying (c) is

$$
\left[\begin{array}{c}
r_{2} \\
r_{2}-1
\end{array}\right]_{q}-\left[\begin{array}{c}
\partial\left(x_{2}, x_{1}\right) \\
\partial\left(x_{2}, x_{1}\right)-1
\end{array}\right]_{q}=\left[\begin{array}{c}
r_{2} \\
1
\end{array}\right]_{q}-\left[\begin{array}{c}
\partial\left(x_{2}, x_{1}\right) \\
1
\end{array}\right]_{q}
$$

Combining this with Lemmas 2.4 and 2.6] we easily get the following expression for $\mathbf{b}_{r_{1}, r_{2}}$, which simplifies the one given in Theorem 2.3

$$
\mathbf{b}_{r_{1}, r_{2}}(t)=\left[\begin{array}{c}
r_{2} \\
1
\end{array}\right]_{q} \frac{q^{n}-q^{r_{1}+t}}{q^{r_{2}-1}(q-1)}-\left[\begin{array}{c}
t \\
1
\end{array}\right]_{q} \frac{q^{n}-q^{r_{1}+t}}{q^{r_{2}-1}(q-1)} .
$$

Lemma 2.7. Given $\left(x_{1}, x_{2}\right) \in \mathrm{X}_{r_{1}} \times \mathrm{X}_{r_{2}},(z, \omega) \in \widetilde{\mathbf{S}}_{1}\left(x_{2}\right)$ and $y=z \oplus \mathbb{K} \omega \in \mathbf{S}_{1}\left(x_{2}\right)$, the following assertions are equivalent:

(a) $\partial\left(y, x_{1}\right)=\partial\left(x_{2}, x_{1}\right)-1$.

(b) $x_{1}+z \neq x_{1}+x_{2}$ and $\omega \in x_{1}+z$.

(c) $x_{1} \cap x_{2} \subset z$ and $\omega \in x_{1}+z$.

Moreover, when them hold we have $\partial\left(x_{1}+z\right)=\partial\left(x_{1}+x_{2}\right)-1$ and $z=\left(x_{1}+z\right) \cap x_{2}$.

Proof. By (8) and the remark after it we deduce the equivalence between (a) and (b). The equivalence between (b) and (c) follows again from the identity

$$
\partial\left(\left(x_{1}+x_{2}\right) /\left(x_{1}+z\right)\right)=1-\partial\left(x_{1} \cap x_{2}\right)+\partial\left(x_{1} \cap z\right) .
$$

Relation $\partial\left(x_{1}+z\right)=\partial\left(x_{1}+x_{2}\right)-1$ is immediate from (b). The last claim follows from the modular law $\left(x_{1}+z\right) \cap x_{2}=\left(x_{1}+z\right) \cap\left(x_{2}+z\right)=\left(x_{1} \cap x_{2}\right)+z=z$.

Finally, arguing as we did after Lemma 2.6. we easily obtain from Lemmas 2.4 and 2.7 the following identity

$$
\mathbf{c}_{r_{1}, r_{2}}(t)=\left[\begin{array}{l}
t \\
1
\end{array}\right]_{q} \frac{q^{r_{1}+t-1}-q^{r_{2}-1}}{q^{r_{2}-1}(q-1)}
$$

This is the identity given in (e) and the proof of Theorem 2.3 is completed.

\section{THE KERNELS IN $\operatorname{Hom}_{\mathrm{G}}\left(\mathrm{V}_{r_{1}, s}, \mathrm{~V}_{r_{2}, s}\right)$}

In this section we obtain explicit formulas for the kernels of the operators $\Lambda_{s}^{r_{1}, r_{2}}$ in terms of the basic hypergeometric function. More concretely, it turns out that these kernels (when regarded as functions of $q^{-t}$ with $t \in \mathrm{I}_{n}\left(r_{1}, r_{2}\right)$ ) are given by the so-called $q$-Hahn polynomials. After that, we shall also provide Rodrigues type formulas for these kernels adapting the techniques developed in [9]. The basic idea consist in showing that the operator $\mathcal{L}_{r_{1}, r_{2}}$ introduced above can be identified with the hypergeometric operator studied in $[9]$.

3.1. Preliminaries. As we have pointed out, we shall need some results from the theory of basic hypergeometric polynomials appearing in 9. However, since the paper 9 considers a great variety of hypergeometric type operators, we summarize and re-state here those results from 9] that will be useful in the sequel. To be more precise, we shall formulate the main results from [9] in the particular case of the (so-called there) geometric canonical form. Let us denote by $\mathbb{P}_{r}[x]$ the space of 
complex polynomials of degree $\leq r$ in one variable. Given a polynomial $f \in \mathbb{P}_{r}[x]$ and any $q \neq 1$, we define the linear operators

$$
\mathrm{S} f(u)=\frac{f\left(q^{1 / 2} u\right)+f\left(q^{-1 / 2} u\right)}{2} \quad \text { and } \quad \mathrm{D} f(u)=\frac{f\left(q^{1 / 2} u\right)-f\left(q^{-1 / 2} u\right)}{\left(q^{1 / 2}-q^{-1 / 2}\right) u} .
$$

It is not difficult to check that $\mathrm{S}: \mathbb{P}_{r}[x] \rightarrow \mathbb{P}_{r}[x]$ and $\mathrm{D}: \mathbb{P}_{r}[x] \rightarrow \mathbb{P}_{r-1}[x]$. Moreover, these operators can be extended so that S, D : $\mathcal{M}\left(\mathbb{C}^{*}\right) \rightarrow \mathcal{M}\left(\mathbb{C}^{*}\right)$, where $\mathcal{M}\left(\mathbb{C}^{*}\right)$ stands for the space of meromorphic functions on $\mathbb{C} \backslash\{0\}$, see [9] Section 2.3]. Now, given $\sigma \in \mathbb{P}_{2}[x]$ and $\tau \in \mathbb{P}_{1}[x]$ by

$$
\sigma(x)=\alpha_{2} x^{2}+\alpha_{1} x+\alpha_{0} \quad \text { and } \quad \tau(x)=\beta_{1} x+\beta_{0},
$$

we consider the hypergeometric operator

$$
\mathrm{L}=\sigma \mathrm{D}^{2}+\tau \mathrm{SD} .
$$

The following result has been adapted from [9] according to our aims.

Lemma 3.1. The hypergeometric operator $\mathrm{L}: \mathbb{P}_{r}[x] \rightarrow \mathbb{P}_{r}[x]$ satisfy:

(a) Let us consider two polynomials

$$
\begin{aligned}
& \chi^{+}(u)=\gamma_{2}^{+} u^{2}+\gamma_{1}^{+} u+\gamma_{0}, \\
& \chi^{-}(u)=\gamma_{2}^{-} u^{2}+\gamma_{1}^{-} u+\gamma_{0},
\end{aligned}
$$

with the same value at $u=0$ and let us parameterize $\sigma$ and $\tau$ as follows

$$
\begin{array}{ll}
\chi^{+}(u)=\sigma(u)+\frac{q^{-1 / 2}-q^{1 / 2}}{2} u \tau(u), & \sigma(u)=\frac{\chi^{+}(u)+\chi^{-}(u)}{2}, \\
\chi^{-}(u)=\sigma(u)-\frac{q^{-1 / 2}-q^{1 / 2}}{2} u \tau(u), & \tau(u)=\frac{\chi^{+}(u)-\chi^{-}(u)}{\left(q^{-1 / 2}-q^{1 / 2}\right) u} .
\end{array}
$$

Assume that the following numbers are pairwise distinct for $0 \leq k \leq r$

$$
\mu_{k}=\frac{q^{k / 2}-q^{-k / 2}}{\left(q^{1 / 2}-q^{-1 / 2}\right)^{2}}\left[\gamma_{2}^{+} q^{(1-k) / 2}-\gamma_{2}^{-} q^{(k-1) / 2}\right] .
$$

Then there exist eigenfunctions $f_{k} \in \mathbb{P}_{k}[x]$ with degree $k$ satisfying

$$
\mathrm{L} f_{k}(u)+\mu_{k} f_{k}(u)=0 .
$$

(b) Let $f_{k}$ be as above and let $\partial_{k} f_{k}$ be the main coefficient of $f_{k}$ :

- Given $u_{0}$ such that $\gamma_{2}^{+} u_{0}^{2}+\gamma_{1}^{+} u_{0}+\gamma_{0}=0$, we have

$$
f_{k}(u)=\partial_{k} f_{k} \sum_{j=0}^{k}\left(\prod_{i=j}^{k-1} \frac{1-q^{-(1+i)}}{q^{1 / 2}\left(1-q^{-1}\right)^{2}} \frac{\chi^{-}\left(q^{i} u_{0}\right)}{\left(\mu_{i}-\mu_{k}\right) u_{0}}\right) \prod_{i=0}^{j-1}\left(u-q^{i} u_{0}\right) \text {. }
$$

- Given $u_{0}$ such that $\gamma_{2}^{-} u_{0}^{2}+\gamma_{1}^{-} u_{0}+\gamma_{0}=0$, we have

$$
f_{k}(u)=\partial_{k} f_{k} \sum_{j=0}^{k}\left(\prod_{i=j}^{k-1} \frac{1-q^{1+i}}{q^{-1 / 2}(q-1)^{2}} \frac{\chi^{+}\left(q^{-i} u_{0}\right)}{\left(\mu_{i}-\mu_{k}\right) u_{0}}\right) \prod_{i=0}^{j-1}\left(u-q^{-i} u_{0}\right) .
$$

(c) Moreover, assuming above that $f_{k}\left(u_{0}\right) \neq 0$, we obtain:

- If $\gamma_{2}^{+} u_{0}^{2}+\gamma_{1}^{+} u_{0}+\gamma_{0}=0$ and $f_{k}\left(u_{0}\right) \neq 0$, we have

$$
f_{k}(u)=f_{k}\left(u_{0}\right) \sum_{j=0}^{k}\left(\prod_{i=0}^{j-1} \frac{q^{1 / 2}\left(1-q^{-1}\right)^{2}}{1-q^{-(1+i)}} \frac{\left(\mu_{i}-\mu_{k}\right) u_{0}}{\chi^{-}\left(q^{i} u_{0}\right)}\right) \prod_{i=0}^{j-1}\left(u-q^{i} u_{0}\right) \text {. }
$$


- If $\gamma_{2}^{-} u_{0}^{2}+\gamma_{1}^{-} u_{0}+\gamma_{0}=0$ and $f_{k}\left(u_{0}\right) \neq 0$, we have

$$
f_{k}(u)=f_{k}\left(u_{0}\right) \sum_{j=0}^{k}\left(\prod_{i=0}^{j-1} \frac{q^{-1 / 2}(q-1)^{2}}{1-q^{1+i}} \frac{\left(\mu_{i}-\mu_{k}\right) u_{0}}{\chi^{+}\left(q^{-i} u_{0}\right)}\right) \prod_{i=0}^{j-1}\left(u-q^{-i} u_{0}\right) \text {. }
$$

(d) If $\chi^{ \pm}(u)=\left(1-\xi_{0}^{ \pm} u\right)\left(1-\xi_{1}^{ \pm} u\right)$, we obtain the $q$-Hahn polynomials

$$
\begin{aligned}
& \text { - } f_{k}(u)=f_{k}\left(1 / \xi_{0}^{+}\right){ }_{3} \phi_{2}\left(\begin{array}{c}
q^{k}, \xi_{0}^{+} u, q^{1-k} \xi_{0}^{+} \xi_{1}^{+} / \xi_{0}^{-} \xi_{1}^{-} \\
\xi_{0}^{+} / \xi_{0}^{-}, \xi_{0}^{+} / \xi_{1}^{-}
\end{array} q^{-1}, q^{-1}\right) \text {. } \\
& \text { - } f_{k}(u)=f_{k}\left(1 / \xi_{1}^{+}\right){ }_{3} \phi_{2}\left(\begin{array}{c}
q^{k}, \xi_{1}^{+} u, q^{1-k} \xi_{0}^{+} \xi_{1}^{+} / \xi_{0}^{-} \xi_{1}^{-} \\
\xi_{1}^{+} / \xi_{0}^{-}, \xi_{1}^{+} / \xi_{1}^{-}
\end{array} q^{-1}, q^{-1}\right) \text {. } \\
& \text { - } f_{k}(u)=f_{k}\left(1 / \xi_{0}^{-}\right){ }_{3} \phi_{2}\left(\begin{array}{c}
q^{k}, 1 / \xi_{0}^{-} u, q^{1-k} \xi_{0}^{+} \xi_{1}^{+} / \xi_{0}^{-} \xi_{1}^{-} \\
\xi_{0}^{+} / \xi_{0}^{-}, \xi_{1}^{+} / \xi_{0}^{-}
\end{array} \mid q^{-1}, q^{-1} \xi_{1}^{-} u\right) \text {. } \\
& \text { - } f_{k}(u)=f_{k}\left(1 / \xi_{1}^{-}\right)_{3} \phi_{2}\left(\begin{array}{c}
q^{k}, 1 / \xi_{1}^{-} u, q^{1-k} \xi_{0}^{+} \xi_{1}^{+} / \xi_{0}^{-} \xi_{1}^{-} \\
\xi_{0}^{+} / \xi_{1}^{-}, \xi_{1}^{+} / \xi_{1}^{-}
\end{array} \mid q^{-1}, q^{-1} \xi_{0}^{-} u\right) \text {. }
\end{aligned}
$$

Remark 3.2. The role of $q$ in this paper is played by $q^{-1}$ in 9 .

Remark 3.3. The notation for the basic hypergeometric function follows [

We shall also give a Rodrigues type formula for the kernels in $\operatorname{Hom}_{\mathrm{G}}\left(\mathrm{V}_{r_{1}, s}, \mathrm{~V}_{r_{2}, s}\right)$. To that aim, we state below the Rodrigues formula given in [9] which corresponds to our problem. That is, the one for the geometric canonical form.

Lemma 3.4. Let $\rho \in \mathcal{M}\left(\mathbb{C}^{*}\right)$ be a function satisfying the functional equation

$$
\rho(u) \chi^{+}(u)=\rho\left(q^{-1} u\right) \chi^{-}\left(q^{-1} u\right) .
$$

Let $\rho_{j} \in \mathcal{M}\left(\mathbb{C}^{*}\right)$, with $j \geq 0$ and $\rho_{0}=\rho$, determined by any of the recurrences

$$
\begin{aligned}
& \rho_{j+1}(u)=\rho_{j}\left(q^{1 / 2} u\right) \chi^{+}\left(q^{-(j-1) / 2} u\right), \\
& \rho_{j+1}(u)=\rho_{j}\left(q^{-1 / 2} u\right) \chi^{-}\left(q^{(j-1) / 2} u\right) .
\end{aligned}
$$

Then, the following Rodrigues formula holds for the eigenfunctions $f_{0}, f_{1}, \ldots, f_{r}$

$$
\left(\prod_{j=0}^{k-1}\left(\mu_{j}-\mu_{k}\right)\right) \rho(u) f_{k}(u)=\partial_{k} f_{k}\left(\prod_{j=0}^{k-1} \frac{q^{(k-j) / 2}-q^{(j-k) / 2}}{q^{1 / 2}-q^{-1 / 2}}\right) \mathrm{D}^{k} \rho_{k}(u) .
$$

3.2. Polynomic expressions. In this paragraph we express the kernels of the operators of $\mathbf{B}$ in terms of the basic hypergeometric function. Let us recall that, given $0 \leq r_{1}, r_{2} \leq n$, the parameter $\mathrm{N}\left(r_{1}, r_{2}\right)$ takes the value

$$
r_{1} \wedge r_{2} \wedge\left(n-r_{1}\right) \wedge\left(n-r_{2}\right)
$$

and the dimension of $\operatorname{Hom}_{\mathrm{G}}\left(\mathrm{V}_{r_{1}}, \mathrm{~V}_{r_{2}}\right)$ is $1+\mathrm{N}\left(r_{1}, r_{2}\right)$. In particular, we can define the linear isomorphism $\Phi: \mathbb{P}_{\mathrm{N}\left(r_{1}, r_{2}\right)} \rightarrow \operatorname{Hom}_{\mathrm{G}}\left(\mathrm{V}_{r_{1}}, \mathrm{~V}_{r_{2}}\right)$ given by

$$
\Lambda \varphi\left(x_{2}\right)=\sum_{x_{1} \in \mathrm{X}_{r_{1}}} f\left(q^{-\partial\left(x_{2}, x_{1}\right)}\right) \varphi\left(x_{1}\right)
$$

for $\Lambda=\Phi f$. In other words, the kernel $\lambda: \mathrm{I}_{n}\left(r_{1}, r_{2}\right) \rightarrow \mathbb{C}$ of $\Phi f$ has the form

$$
\lambda(t)=f\left(q^{-t}\right) .
$$

Then we define $\mathrm{L}_{r_{1}, r_{2}}: \mathbb{P}_{\mathrm{N}\left(r_{1}, r_{2}\right)} \rightarrow \mathbb{P}_{\mathrm{N}\left(r_{1}, r_{2}\right)}$ by the relation $\mathrm{L}_{r_{1}, r_{2}}=\Phi^{-1} \circ \mathcal{L}_{r_{1}, r_{2}} \circ \Phi$. 
Lemma 3.5. Let us consider the following polynomials in $\mathbb{P}_{2}[x]$

$$
\begin{aligned}
& \chi^{+}(u)=q^{r_{1}-r_{2}-1 / 2}\left(1-q^{r_{2}} u\right)\left(1-q^{n-r_{1}} u\right), \\
& \chi^{-}(u)=q^{r_{1}-r_{2}-1 / 2}(1-u)\left(1-q^{r_{2}-r_{1}} u\right),
\end{aligned}
$$

and let us parameterize $\sigma \in \mathbb{P}_{2}[x]$ and $\tau \in \mathbb{P}_{1}[x]$ as follows

$$
\begin{aligned}
\chi^{+}(u) & =\sigma(u)+\frac{q^{-1 / 2}-q^{1 / 2}}{2} u \tau(u), & \sigma(u) & =\frac{\chi^{+}(u)+\chi^{-}(u)}{2}, \\
\chi^{-}(u) & =\sigma(u)-\frac{q^{-1 / 2}-q^{1 / 2}}{2} u \tau(u), & \tau(u) & =\frac{\chi^{+}(u)-\chi^{-}(u)}{\left(q^{-1 / 2}-q^{1 / 2}\right) u} .
\end{aligned}
$$

Then, if $\mathrm{S}$ and $\mathrm{D}$ stand for the operators defined above, we have

$$
\mathrm{L}_{r_{1}, r_{2}}=\sigma \mathrm{D}^{2}+\tau \mathrm{SD} .
$$

Proof. Given $f \in \mathbb{P}_{\mathrm{N}\left(r_{1}, r_{2}\right)}$, we have

$$
\begin{aligned}
& \sigma \mathrm{D}^{2} f\left(q^{-t}\right)+\tau \operatorname{SD} f\left(q^{-t}\right) \\
& =\frac{\sigma\left(q^{-t}\right)}{q^{-t-1 / 2}-q^{-t+1 / 2}}\left[\frac{f\left(q^{-t-1}\right)-f\left(q^{-t}\right)}{q^{-t-1}-q^{-t}}-\frac{f\left(q^{-t}\right)-f\left(q^{-t+1}\right)}{q^{-t}-q^{-t+1}}\right] \\
& +\frac{\tau\left(q^{-t}\right)}{2}\left[\frac{f\left(q^{-t-1}\right)-f\left(q^{-t}\right)}{q^{-t-1}-q^{-t}}+\frac{f\left(q^{-t}\right)-f\left(q^{-t+1}\right)}{q^{-t}-q^{-t+1}}\right] \\
& \quad=\frac{2 \sigma\left(q^{-t}\right)+\left(q^{-1 / 2}-q^{1 / 2}\right) q^{-t} \tau\left(q^{-t}\right)}{2(q-1)^{2} q^{-2 t-3 / 2}}(\lambda(t+1)-\lambda(t)) \\
& +\frac{2 \sigma\left(q^{-t}\right)-\left(q^{-1 / 2}-q^{1 / 2}\right) q^{-t} \tau\left(q^{-t}\right)}{2(q-1)^{2} q^{-2 t-1 / 2}}(\lambda(t-1)-\lambda(t)) \\
& =\frac{q^{2 t+3 / 2} \chi^{+}\left(q^{-t}\right)}{(q-1)^{2}}(\lambda(t+1)-\lambda(t))+\frac{q^{2 t+1 / 2} \chi^{-}\left(q^{-t}\right)}{(q-1)^{2}}(\lambda(t-1)-\lambda(t)) .
\end{aligned}
$$

Now, applying Theorem 2.3 the last expression equals

$$
\mathbf{b}_{r_{1}, r_{2}}(t)(\lambda(t+1)-\lambda(t))+\mathbf{c}_{r_{1}, r_{2}}(t)(\lambda(t-1)-\lambda(t)) .
$$

Applying again Theorem 2.3] the proof is concluded by the definition of $\mathrm{L}_{r_{1}, r_{2}}$.

Theorem 3.6. The spaces $\mathrm{V}_{r, s}$ are $\mathcal{L}_{r}$-eigenspaces with eigenvalue $-\mu_{s}(n)$, where

$$
\mu_{s}(n)=\left(q^{s}-1\right) \frac{q^{n-s+1}-1}{(q-1)^{2}} .
$$

Moreover, the operators $\Lambda_{s}^{r_{1}, r_{2}} \in \mathbf{B}$ satisfy

$$
\mathcal{L}_{r_{1}, r_{2}} \Lambda_{s}^{r_{1}, r_{2}}+\mu_{s}(n) \Lambda_{s}^{r_{1}, r_{2}}=0 .
$$

On the other hand, if we consider the polynomials $f_{s}^{r_{1}, r_{2}} \in \mathbb{P}_{s}[x]$ determined by

$$
f_{s}^{r_{1}, r_{2}}\left(q^{-t}\right)=\lambda_{s}^{r_{1}, r_{2}}(t)
$$

then $f_{s}^{r_{1}, r_{2}}$ is a q-Hahn polynomial of degree s given by

$\left(\mathrm{H}_{q}(1)\right) f_{s}^{r_{1}, r_{2}}(u)=f_{s}^{r_{1}, r_{2}}(1){ }_{3} \phi_{2}\left(\begin{array}{c}q^{s}, u^{-1}, q^{n-s+1} \\ q^{n-r_{1}}, q^{r_{2}}\end{array} \mid q^{-1}, q^{r_{2}-r_{1}-1} u\right)$.
$\left(\mathrm{H}_{q}(2)\right) f_{s}^{r_{1}, r_{2}}(u)=f_{s}^{r_{1}, r_{2}}\left(q^{-r_{2}}\right){ }_{3} \phi_{2}\left(\begin{array}{c}q^{s}, q^{r_{2}} u, q^{n-s+1} \\ q^{r_{1}}, q^{r_{2}}\end{array} \mid q^{-1}, q^{-1}\right)$.
$\left(\mathrm{H}_{q}(3)\right) f_{s}^{r_{1}, r_{2}}(u)=f_{s}^{r_{1}, r_{2}}\left(q^{r_{1}-n}\right){ }_{3} \phi_{2}\left(\begin{array}{c}q^{s}, q^{n-r_{1}} u, q^{n-s+1} \\ q^{n-r_{1}}, q^{n-r_{2}}\end{array} \mid q^{-1}, q^{-1}\right)$. 
$\left(\mathrm{H}_{q}(4)\right) f_{s}^{r_{1}, r_{2}}(u)=f_{s}^{r_{1}, r_{2}}\left(q^{r_{1}-r_{2}}\right)_{3} \phi_{2}\left(\begin{array}{c}q^{s}, q^{r_{1}-r_{2}} u^{-1}, q^{n-s+1} \\ q^{r_{1}}, q^{n-r_{2}}\end{array} \mid q^{-1}, q^{-1} u\right)$.

Proof. Notice that $\mu_{s}(n)$ are pairwise distinct for $0 \leq s \leq \mathrm{N}\left(r_{1}, r_{2}\right)$, since

$$
\mu_{s_{1}}(n)-\mu_{s_{2}}(n)=q^{s_{2}}\left(q^{s_{1}-s_{2}}-1\right) \frac{q^{n+1-s_{1}-s_{2}}-1}{(q-1)^{2}} .
$$

Therefore, we know from Lemmas 3.1 and 3.5 that

$$
\left\{\left(1-q^{s}\right) \frac{q^{n-s+1}-1}{(q-1)^{2}} \mid 0 \leq s \leq \mathrm{N}\left(r_{1}, r_{2}\right)\right\}
$$

is the family of eigenvalues of $\mathrm{L}_{r_{1}, r_{2}}$. In particular, it turns out that this family is the family of eigenvalues of $\mathcal{L}_{r}$ when $0 \leq s \leq r \wedge(n-r)$ and of $\mathcal{L}_{r_{1}, r_{2}}$ when $0 \leq s \leq \mathrm{N}\left(r_{1}, r_{2}\right)$. By Theorem 2.3 we deduce that, for any $0 \leq r \leq[n / 2]-1$, all the eigenvalues of $\mathcal{L}_{r}$ are eigenvalues of $\mathcal{L}_{r+1}$ and the operator $\mathcal{L}_{r+1}$ has one more eigenvalue associated to the eigenspace $\mathrm{V}_{r+1, r+1}$. Applying a simple induction argument, we know that the eigenvalue $-\mu_{s}(n)$ of $\mathcal{L}_{r}$ is associated to the eigenspace $\mathrm{V}_{r, s}$. As a particular case, we obtain the relation

$$
\mathcal{L}_{r_{1}, r_{2}} \Lambda_{s}^{r_{1}, r_{2}}+\mu_{s}(n) \Lambda_{s}^{r_{1}, r_{2}}=0 .
$$

Once we have identified the eigenvalue corresponding to the operator $\Lambda_{s}^{r_{1}, r_{2}}$, the given expressions for the polynomial $f_{s}^{r_{1}, r_{2}}$ in terms of the $q$-Hahn polynomials follow easily from Lemma 3.1 This completes the proof.

Remark 3.7. Let us denote by $\partial_{s} f_{s}^{r_{1}, r_{2}}$ the main coefficient of $f_{s}^{r_{1}, r_{2}}$. Then, by looking at the main coefficients of the expressions given in Theorem 3.6] it is not difficult to check that the following relations hold

$$
\begin{aligned}
f_{s}^{r_{1}, r_{2}}(1) & =\partial_{s} f_{s}^{r_{1}, r_{2}}(-1)^{s} q^{s\left(r_{1}-r_{2}\right)-\left(\begin{array}{c}
s \\
2
\end{array}\right)} \frac{\left(q^{n-r_{1}}, q^{r_{2}} ; q^{-1}\right)_{s}}{\left(q^{n-s+1} ; q^{-1}\right)_{s}}, \\
f_{s}^{r_{1}, r_{2}}\left(q^{-r_{2}}\right) & =\partial_{s} f_{s}^{r_{1}, r_{2}} q^{-s r_{2}} \frac{\left(q^{r_{1}}, q^{r_{2}} ; q^{-1}\right)_{s}}{\left(q^{n-s+1} ; q^{-1}\right)_{s}} \\
f_{s}^{r_{1}, r_{2}}\left(q^{r_{1}-n}\right) & =\partial_{s} f_{s}^{r_{1}, r_{2}} q^{-s\left(n-r_{1}\right)} \frac{\left(q^{n-r_{1}}, q^{n-r_{2}} ; q^{-1}\right)_{s}}{\left(q^{n-s+1} ; q^{-1}\right)_{s}} . \\
f_{s}^{r_{1}, r_{2}}\left(q^{r_{1}-r_{2}}\right) & =\partial_{s} f_{s}^{r_{1}, r_{2}}(-1)^{s} q^{-\left(\begin{array}{c}
s \\
2
\end{array}\right)} \frac{\left(q^{r_{1}}, q^{n-r_{2}} ; q^{-1}\right)_{s}}{\left(q^{n-s+1} ; q^{-1}\right)_{s}} .
\end{aligned}
$$

Remark 3.8. The basic hypergeometric series in Theorem 3.6 must be truncated at degree $s$. That is, the terms of degree $>s$ must be ignored. This is a consequence of the term $q^{s}$ which appears in any of them. For instance, we have

$$
\begin{aligned}
f_{s}^{r, s}(u) & =f_{s}^{r, s}(1){ }_{3} \phi_{2}\left(\begin{array}{c}
q^{s}, u^{-1}, q^{n-s+1} \\
q^{n-r}, q^{s}
\end{array} \mid q^{-1}, q^{s-r-1} u\right) \\
& =f_{s}^{r, s}(1) \sum_{k=0}^{s} \frac{\left(u^{-1}, q^{n-s+1} ; q^{-1}\right)_{k}}{\left(q^{-1}, q^{n-r} ; q^{-1}\right)_{k}} q^{k(s-r-1)} u^{k},
\end{aligned}
$$

which is a truncated ${ }_{2} \phi_{1}$ series. Now, evaluating at $u=q^{-t}$ for some $t \in \mathrm{I}_{n}(r, s)$ and applying the $q$-Gauss summation formula, we easily obtain

$$
f_{s}^{r, s}\left(q^{-t}\right)=f_{s}^{r, s}(1) \frac{\left(q^{s-r-1} ; q^{-1}\right)_{t}}{\left(q^{n-r} ; q^{-1}\right)_{t}} .
$$


Similarly, we have for $t \in \mathrm{I}_{n}(n-s, r)$

$$
f_{s}^{n-s, r}\left(q^{-t}\right)=f_{s}^{n-s, r}(1) \frac{\left(q^{r+s-n-1} ; q^{-1}\right)_{t}}{\left(q^{r} ; q^{-1}\right)_{t}} .
$$

At this point, the identity

$$
\frac{f_{s}^{r_{1}, r_{2}}(u)}{f_{s}^{r_{1}, r_{2}}(1)}=q^{s\left(r_{2}-r_{1}\right)} \frac{\left(q^{r_{1}}, q^{n-r_{2}} ; q^{-1}\right)_{s}}{\left(q^{n-r_{1}}, q^{r_{2}} ; q^{-1}\right)_{s}} \frac{f_{s}^{r_{2}, r_{1}}\left(q^{r_{2}-r_{1}} u\right)}{f_{s}^{r_{2}, r_{1}}(1)}
$$

follows from certain transformation formulas for the basic hypergeometric series ${ }_{3} \phi_{2}$, see [1. In Section [5] we shall provide an alternative (combinatorial) proof of this identity. Therefore, we prefer to omit the details of the proof just sketched.

3.3. Rodrigues formula. In this paragraph we provide a Rodrigues type formula for the kernels $\lambda_{s}^{r_{1}, r_{2}}$ of the operators in $\mathbf{B}$. More concretely, given any two integers $0 \leq r_{1}, r_{2} \leq n$ and $0 \leq s \leq \mathrm{N}\left(r_{1}, r_{2}\right)$, we shall study the eigenfunctions $f_{s}^{r_{1}, r_{2}}$ defined above by the relation

$$
f_{s}^{r_{1}, r_{2}}\left(q^{-t}\right)=\lambda_{s}^{r_{1}, r_{2}}(t) .
$$

As it was noticed in 9], the Rodrigues formula provided by Lemma 3.4 is not unique since the given functional equation has multiple solutions. Hence, the main difficulty will be to choose the right solution of the functional equation according to our further purposes. Following Lemma 3.4 let us consider $\rho^{r_{1}, r_{2}} \in \mathcal{M}\left(\mathbb{C}^{*}\right)$ satisfying the functional equation

$$
\rho^{r_{1}, r_{2}}(u) \chi^{+}(u)=\rho^{r_{1}, r_{2}}\left(q^{-1} u\right) \chi^{-}\left(q^{-1} u\right),
$$

with $\chi^{+}$and $\chi^{-}$determined by Lemma [3.5 Moreover, let

$$
\left\{\rho_{s}^{r_{1}, r_{2}} \mid 0 \leq s \leq \mathrm{N}\left(r_{1}, r_{2}\right)\right\}
$$

be the family of functions in $\mathcal{M}\left(\mathbb{C}^{*}\right)$ defined by any of the recurrences

$$
\begin{aligned}
& \rho_{s+1}^{r_{1}, r_{2}}(u)=\rho_{s}^{r_{1}, r_{2}}\left(q^{1 / 2} u\right) \chi^{+}\left(q^{-(s-1) / 2} u\right), \\
& \rho_{s+1}^{r_{1}, r_{2}}(u)=\rho_{s}^{r_{1}, r_{2}}\left(q^{-1 / 2} u\right) \chi^{-}\left(q^{(s-1) / 2} u\right),
\end{aligned}
$$

where $\rho_{0}^{r_{1}, r_{2}}=\rho^{r_{1}, r_{2}}$. Then, implementing in Lemma 3.4 the eigenvalues provided by Theorem 3.6 it is not difficult to see that we obtain the following Rodrigues formula for the eigenfunctions $f_{s}^{r_{1}, r_{2}}$

$$
\left(\mathrm{R}_{s}\left(r_{1}, r_{2}\right)\right) \quad \rho^{r_{1}, r_{2}}(u) f_{s}^{r_{1}, r_{2}}(u)=\partial_{s} f_{s}^{r_{1}, r_{2}} q^{-\frac{3}{2}\left(\begin{array}{c}
s \\
2
\end{array}\right)} \frac{(q-1)^{s}}{\left(q^{n-s+1} ; q^{-1}\right)_{s}} \mathrm{D}^{s} \rho_{s}^{r_{1}, r_{2}}(u) .
$$

Remark 3.9. Following Section 4.5 of $[9$, the easiest solution to the functional equation (9) and the recurrences (10) is given by

$$
\begin{aligned}
& \varrho^{r_{1}, r_{2}}(u)=\frac{\left(q^{-1} u, q^{r_{2}-r_{1}-1} u ; q^{-1}\right)_{\infty}}{\left(q^{r_{2}} u, q^{n-r_{1}} u ; q^{-1}\right)_{\infty}}, \\
& \varrho_{s}^{r_{1}, r_{2}}(u)=q^{s\left(r_{1}-r_{2}-1 / 2\right)} \frac{\left(q^{-1+s / 2} u, q^{r_{2}-r_{1}-1+s / 2} u ; q^{-1}\right)_{\infty}}{\left(q^{r_{2}-s / 2} u, q^{n-r_{1}-s / 2} u ; q^{-1}\right)_{\infty}} .
\end{aligned}
$$

Although the system of functions given in Remark 3.9 provides the simplest Rodrigues formula for the eigenfunctions $f_{s}^{r_{1}, r_{2}}$, it is not the most appropriate for our aims. Namely, let us analyze the singularities of the function $\varrho^{r_{1}, r_{2}}$. If we take $u_{0}=q^{-t}$ for some $t \in \mathbb{Z}$, then the function $\varrho^{r_{1}, r_{2}}$ has: 
- A double pole at $u_{0}$ when

$$
0 \vee\left(r_{2}-r_{1}\right) \leq t \leq r_{2} \wedge\left(n-r_{1}\right) .
$$

- A simple pole at $u_{0}$ when

$$
\begin{aligned}
& 0 \wedge\left(r_{2}-r_{1}\right) \leq t<0 \vee\left(r_{2}-r_{1}\right), \\
& r_{2} \wedge\left(n-r_{1}\right)<t \leq r_{2} \vee\left(n-r_{1}\right) .
\end{aligned}
$$

- A non-vanishing regular point at $u_{0}$ when

$$
\begin{aligned}
& t<0 \wedge\left(r_{2}-r_{1}\right), \\
& t>r_{2} \vee\left(n-r_{1}\right) .
\end{aligned}
$$

The function $\varrho^{r_{1}, r_{2}}$ does not have any other zeros or poles in $\mathbb{C} \backslash\{0\}$. In particular, it turns out that the function $\varrho^{r_{1}, r_{2}}$ has singular points at $q^{-t}$ with $t$ belonging to the domain

$\mathrm{I}_{n}\left(r_{1}, r_{2}\right)=\left\{\partial\left(x_{2}, x_{1}\right) \mid x_{1} \in \mathrm{X}_{r_{1}}, x_{2} \in \mathrm{X}_{r_{2}}\right\}=\left\{0 \vee\left(r_{2}-r_{1}\right) \leq t \leq r_{2} \wedge\left(n-r_{1}\right)\right\}$.

Obviously, if we want to apply Rodrigues formula $\left.\mathrm{R}_{s}\left(r_{1}, r_{2}\right)\right]$, we need regular solutions of the functional equation (9) at $q^{-t}$ for $t \in \mathrm{I}_{n}\left(r_{1}, r_{2}\right)$. Any other system of solutions can be constructed by taking

$$
\rho^{r_{1}, r_{2}}(u)=k(u) \varrho^{r_{1}, r_{2}}(u),
$$

with $k$ being meromorphic in $\mathbb{C} \backslash\{0\}$ and satisfying $k(u)=k(q u)$. Then, the functions $\rho_{s}^{r_{1}, r_{2}}$ arise from $\rho^{r_{1}, r_{2}}$ by the recurrences (10). The choice of such a function $k$ is equivalent to the choice of an elliptic function $\mathrm{E}(z)=k\left(e^{2 \pi i z}\right)$ with periods 1 and

$$
\omega=\frac{1}{2 \pi i} \log q
$$

see [9] for further details. Hence, we need to find a function $k \in \mathcal{M}\left(\mathbb{C}^{*}\right)$ satisfying $k(u)=k(q u)$ and having double zeros at $\mathrm{I}_{n}\left(r_{1}, r_{2}\right)$. To that aim, we consider two complex numbers $\xi$ and $\eta$ satisfying the conditions

i) The product $\xi \eta$ equals $q^{n-r_{1}+r_{2}}$.

ii) Both $\xi$ and $\eta$ are not of the form $q^{-t}$ for some integer $t$.

Then, the function

$$
k_{r_{1}, r_{2}}(u)=\alpha_{r_{1}, r_{2}} \frac{\left(q^{r_{2}} u, q^{n-r_{1}} u ; q^{-1}\right)_{\infty}}{\left(\xi u, \eta u ; q^{-1}\right)_{\infty}} \frac{\left(q^{-r_{2}-1} u^{-1}, q^{-n+r_{1}-1} u^{-1} ; q^{-1}\right)_{\infty}}{\left(q^{-1} \xi^{-1} u^{-1}, q^{-1} \eta^{-1} u^{-1} ; q^{-1}\right)_{\infty}}
$$

satisfies the required properties. Indeed, the condition $k(u)=k(q u)$ can be easily checked with the aid of property i). On the other hand, it follows from property ii) that $k$ has no poles in $\mathbb{C} \backslash\{0\}$. Therefore, $q^{-t}$ is a double zero of $k$ for any integer $t$ and $k$ has no other zeros. In particular, the function

$$
\rho^{r_{1}, r_{2}}=k_{r_{1}, r_{2}} \varrho^{r_{1}, r_{2}}
$$

is regular in $\mathbb{C} \backslash\{0\}$, non-vanishing in $\mathrm{I}_{n}\left(r_{1}, r_{2}\right)$ and only vanishes in $q^{-\left(\mathbb{Z} \backslash \mathrm{I}_{n}\left(r_{1}, r_{2}\right)\right)}$.

Remark 3.10. Notice that $\rho^{r_{1}, r_{2}}$ is determined up to a constant $\alpha_{r_{1}, r_{2}}$.

Theorem 3.11. Given $0 \leq r_{1}, r_{2} \leq n$, there exists a family of functions

$$
\left\{\rho_{s}^{r_{1}, r_{2}} \mid 0 \leq s \leq \mathrm{N}\left(r_{1}, r_{2}\right)\right\}
$$

in $\mathcal{M}\left(\mathbb{C}^{*}\right)$ satisfying the following properties: 
(a) The function $\rho^{r_{1}, r_{2}}=\rho_{0}^{r_{1}, r_{2}}$ solves the functional equation (9).

(b) The function $\rho^{r_{1}, r_{2}}$ is regular in $\mathbb{C} \backslash\{0\}$ and vanishes in

$$
q^{-\left(\mathbb{Z} \backslash \mathrm{I}_{n}\left(r_{1}, r_{2}\right)\right)} .
$$

(c) Given any integer $t \in \mathrm{I}_{n}\left(r_{1}, r_{2}\right)$, we have

$$
\rho^{r_{1}, r_{2}}\left(q^{-t}\right)=q^{t\left(r_{1}-r_{2}+t+1\right)}\left[\begin{array}{c}
n \\
t, r_{2}-t, r_{1}-r_{2}+t, n-r_{1}-t
\end{array}\right]_{q} .
$$

(d) Each function $\rho_{s}^{r_{1}, r_{2}}$ arise from $\rho^{r_{1}, r_{2}}$ and the recurrences (10).

(e) Each function $\rho_{s}^{r_{1}, r_{2}}$ is regular in $\mathbb{C} \backslash\{0\}$ and vanishes in

$$
q^{-\left(\mathbb{Z} \backslash \mathrm{I}_{n-2 s}\left(r_{1}-s, r_{2}-s\right)\right)-s / 2} .
$$

(f) Given any integer $t \in \mathrm{I}_{n-2 s}\left(r_{1}-s, r_{2}-s\right)$, we have

$$
\begin{aligned}
& \rho_{s}^{r_{1}, r_{2}}\left(q^{-t-s / 2}\right)=\psi_{s}^{r_{1}, r_{2}}(t)\left[\begin{array}{c}
n-2 s \\
t, r_{2}-s-t, r_{1}-r_{2}+t, n-r_{1}-s-t
\end{array}\right]_{q}, \\
& \text { with } \psi_{s}^{r_{1}, r_{2}} \text { given by } \\
& \psi_{s}^{r_{1}, r_{2}}(t)=q^{s\left(r_{1}-r_{2}-1 / 2\right)+t\left(r_{1}-r_{2}+t+1\right)}\left(q^{n} ; q^{-1}\right)_{2 s} .
\end{aligned}
$$

Proof. Our choice for $\rho^{r_{1}, r_{2}}$ will be $k_{r_{1}, r_{2}} \varrho^{r_{1}, r_{2}}$ with $k_{r_{1}, r_{2}}$ given by (11) and the constant $\alpha_{r_{1}, r_{2}}$ to be fixed. Properties (a) and (b) have already been justified. To prove (c), we observe that the functional equation (9) can be rewritten as

$$
\rho^{r_{1}, r_{2}}\left(q^{-t}\right)=\rho^{r_{1}, r_{2}}\left(q^{-t-1}\right) \frac{\chi^{-}\left(q^{-t-1}\right)}{\chi^{+}\left(q^{-t}\right)},
$$

when $t$ and $t+1$ belong to $\mathrm{I}_{n}\left(r_{1}, r_{2}\right)$. Therefore, if we see that the function

$$
\gamma^{r_{1}, r_{2}}\left(q^{-t}\right)=q^{t\left(r_{1}-r_{2}+t+1\right)}\left[\begin{array}{c}
n \\
t, r_{2}-t, r_{1}-r_{2}+t, n-r_{1}-t
\end{array}\right]_{q}
$$

satisfies (12), we will have $\rho^{r_{1}, r_{2}}\left(q^{-t}\right)=\beta_{r_{1}, r_{2}} \gamma^{r_{1}, r_{2}}\left(q^{-t}\right)$ for any $t \in \mathrm{I}_{n}\left(r_{1}, r_{2}\right)$ and some constant $\beta_{r_{1}, r_{2}}$. Then, property (c) follows by taking the appropriate constant $\alpha_{r_{1}, r_{2}}$. Let us show that $\gamma^{r_{1}, r_{2}}$ satisfies the functional equation (12)

$$
\begin{aligned}
\gamma^{r_{1}, r_{2}}\left(q^{-t}\right) & =\frac{q^{t\left(r_{1}-r_{2}+t+1\right)}(q ; q)_{n}}{(q ; q)_{t}(q ; q)_{r_{2}-t}(q ; q)_{r_{1}-r_{2}+t}(q ; q)_{n-r_{1}-t}} \\
& =\gamma^{r_{1}, r_{2}}\left(q^{-t-1}\right) q^{-\left(r_{1}-r_{2}+2 t+2\right)} \frac{\left(1-q^{t+1}\right)\left(1-q^{r_{1}-r_{2}+t+1}\right)}{\left(1-q^{r_{2}-t}\right)\left(1-q^{n-r_{1}-t}\right)} \\
& =\gamma^{r_{1}, r_{2}}\left(q^{-t-1}\right) \frac{\left(1-q^{-t-1}\right)\left(1-q^{r_{2}-r_{1}-t-1}\right)}{\left(1-q^{r_{2}-t}\right)\left(1-q^{n-r_{1}-t}\right)} \\
& =\gamma^{r_{1}, r_{2}}\left(q^{-t-1}\right) \frac{\chi^{-}\left(q^{-t-1}\right)}{\chi^{+}\left(q^{-t}\right)} .
\end{aligned}
$$

Now the constant $\alpha_{r_{1}, r_{2}}$ is already fixed so that the functions $\rho_{s}^{r_{1}, r_{2}}$ are completely determined by property (d). Taking $u=q^{-t-s / 2}$ in the first recurrence in (10), we obtain the following relation

$$
\rho_{s}^{r_{1}, r_{2}}\left(q^{-t-s / 2}\right)=\rho_{s-1}^{r_{1}, r_{2}}\left(q^{-t-(s-1) / 2}\right) \chi^{+}\left(q^{-s+1-t}\right) .
$$

Property (e) means that

$$
\rho_{s}^{r_{1}, r_{2}}\left(q^{-t-s / 2}\right)=0 \quad \text { for } \quad t \in \mathbb{Z} \backslash \mathrm{I}_{n-2 s}\left(r_{1}-s, r_{2}-s\right) .
$$


This follows easily from the recurrence (13). Indeed, we just need to observe which are the zeros of $\chi^{+}$and apply (b), we leave the details to the reader. Therefore, it remains to see (f). Let us consider the functions

$$
\gamma_{s}^{r_{1}, r_{2}}\left(q^{-t-s / 2}\right)=\psi_{s}^{r_{1}, r_{2}}(t)\left[\begin{array}{c}
n-2 s \\
t, r_{2}-s-t, r_{1}-r_{2}+t, n-r_{1}-s-t
\end{array}\right]_{q} .
$$

It is not difficult to check that each function $\gamma_{s}^{r_{1}, r_{2}}$ arise from $\gamma_{s-1}^{r_{1}, r_{2}}$ and (13). Therefore, property (f) follows from (c) and a simple induction argument.

Remark 3.12. In Theorem 3.11 we have chosen the appropriate solutions of the functional equation (9) and the recurrences (10) for our further purposes. This can be justified by the following combinatorial meaning of these functions. First, by identity (5), we have

$$
\rho^{r_{1}, r_{2}}\left(q^{-t}\right) q^{-t}=\left|\left\{\left(x_{1}, x_{2}\right) \in \mathrm{X}_{r_{1}} \times \mathrm{X}_{r_{2}} \mid \partial\left(x_{2}, x_{1}\right)=t\right\}\right| .
$$

In particular, (2) gives

$$
\sum_{t \in \mathbb{Z}} \rho^{r_{1}, r_{2}}\left(q^{-t}\right) q^{-t}=\left[\begin{array}{c}
n \\
r_{1}
\end{array}\right]_{q}\left[\begin{array}{c}
n \\
r_{2}
\end{array}\right]_{q} .
$$

Second, if $\mathrm{X}_{r}^{n-2 s}$ denotes the set of $r$-dimensional of an $(n-2 s)$-dimensional vector space $\Omega_{s}$ over $\mathbb{K}$, we also have

$$
\rho_{s}^{r_{1}, r_{2}}\left(q^{-t-s / 2}\right) q^{-t}=\delta_{s}^{r_{1}, r_{2}}\left|\left\{\left(x_{1}, x_{2}\right) \in \mathrm{X}_{r_{1}-s}^{n-2 s} \times \mathrm{X}_{r_{2}-s}^{n-2 s} \mid \partial\left(x_{2}, x_{1}\right)=t\right\}\right| .
$$

with $\delta_{s}^{r_{1}, r_{2}}=q^{s\left(r_{1}-r_{2}-1 / 2\right)}\left(q^{n} ; q^{-1}\right)_{2 s}$. In particular,

$$
\begin{aligned}
& \sum_{t \in \mathbb{Z}+\frac{s}{2}} \rho_{s}^{r_{1}, r_{2}}\left(q^{-t}\right) q^{-t}=q^{-s / 2} \sum_{t \in \mathbb{Z}} \rho_{s}^{r_{1}, r_{2}}\left(q^{-t-s / 2}\right) q^{-t} \\
& =q^{s\left(r_{1}-r_{2}-1\right)}\left(q^{n} ; q^{-1}\right)_{2 s}\left[\begin{array}{c}
n-2 s \\
r_{1}-s
\end{array}\right]_{q}\left[\begin{array}{c}
n-2 s \\
r_{2}-s
\end{array}\right]_{q} \\
& =\frac{\left(q^{r_{1}}, q^{n-r_{1}}, q^{r_{2}}, q^{n-r_{2}} ; q^{-1}\right)_{s}}{q^{s\left(r_{2}-r_{1}+1\right)}\left(q^{n} ; q^{-1}\right)_{2 s}}\left[\begin{array}{c}
n \\
r_{1}
\end{array}\right]_{q}\left[\begin{array}{c}
n \\
r_{2}
\end{array}\right]_{q} .
\end{aligned}
$$

Remark 3.13. Our choice in Theorem 3.11 has also the following interpretation. The operator $\mathcal{L}_{r_{1}, r_{2}}$ is clearly self-adjoint with respect to the Hilbert-Schmidt inner product on $\operatorname{Hom}_{\mathrm{G}}\left(\mathrm{V}_{r_{1}}, \mathrm{~V}_{r_{2}}\right)$. In particular, this property can be rewritten in terms of the operator $\mathrm{L}_{r_{1}, r_{2}}$ via the mapping $\Phi: \mathbb{P}_{\mathrm{N}\left(r_{1}, r_{2}\right)} \rightarrow \operatorname{Hom}_{\mathrm{G}}\left(\mathrm{V}_{r_{1}}, \mathrm{~V}_{r_{2}}\right)$. Then, it can be checked that the hypergeometric operator $\mathrm{L}_{r_{1}, r_{2}}$ becomes self-adjoint with respect to the inner product

$$
\langle f, g\rangle=\sum_{t \in \mathrm{I}_{n}\left(r_{1}, r_{2}\right)} \rho^{r_{1}, r_{2}}\left(q^{-t}\right) q^{-t} f\left(q^{-t}\right) \overline{g\left(q^{-t}\right)} .
$$

The role of the factor $q^{-t}$ in this expression will become clear in Lemma 4.3 below. The reader is referred to Section 3.3 of [9] for more on these orthogonality relations.

\section{The Product Formula}

In this section we study a product formula for the operators $\Lambda_{s}^{r_{1}, r_{2}}$ in $\mathbf{B}$. To that aim, our first task is to normalize these operators since there are only determined up to a constant factor. We choose the normalization provided by

$$
f_{s}^{r_{1}, r_{2}}(1)=\lambda_{s}^{r_{1}, r_{2}}(0)=1 .
$$


Following Remark 3.7 we have

$$
\partial_{s} f_{s}^{r_{1}, r_{2}}=(-1)^{s} q^{s\left(r_{2}-r_{1}\right)+\left(\begin{array}{c}
s \\
2
\end{array}\right)} \frac{\left(q^{n-s+1} ; q^{-1}\right)_{s}}{\left(q^{n-r_{1}}, q^{r_{2}} ; q^{-1}\right)_{s}} .
$$

In particular, Rodrigues formula $\mathrm{R}_{s}\left(r_{1}, r_{2}\right)$ becomes

$$
\rho^{r_{1}, r_{2}}(u) f_{s}^{r_{1}, r_{2}}(u)=(-1)^{s} q^{s\left(r_{2}-r_{1}\right)-\frac{1}{2}\left(\begin{array}{c}
s \\
2
\end{array}\right)} \frac{(q-1)^{s}}{\left(q^{n-r_{1}}, q^{r_{2}} ; q^{-1}\right)_{s}} \mathrm{D}^{s} \rho_{s}^{r_{1}, r_{2}}(u) .
$$

Since the representations of $\mathrm{G}$ into the spaces $\mathrm{V}_{r_{1}, s_{1}}$ and $\mathrm{V}_{r_{2}, s_{2}}$ are equivalent if and only if $s_{1}=s_{2}$, we clearly have

$$
\Lambda_{s_{2}}^{r_{3}, r_{4}} \circ \Lambda_{s_{1}}^{r_{1}, r_{2}}=0
$$

unless $s_{1}=s_{2}$ and $r_{2}=r_{3}$. In particular, in order to give an explicit formula for the product of two operators in $\operatorname{End}_{\mathrm{G}}(\mathrm{V})$, it suffices to study the products $\Lambda_{s}^{r_{2}, r_{3}} \circ \Lambda_{s}^{r_{1}, r_{2}}$. In the following result, we assume by convention that

$$
\left[\begin{array}{c}
n \\
-1
\end{array}\right]_{q}=0
$$

Theorem 4.1. If $0 \leq r_{1}, r_{2}, r_{3} \leq n$ and $0 \leq s \leq \mathrm{N}\left(r_{1}, r_{2}\right) \wedge \mathrm{N}\left(r_{2}, r_{3}\right)$, we have

$$
\left(\mathrm{P}_{s}\left(r_{1}, r_{2}, r_{3}\right)\right) \quad \Lambda_{s}^{r_{2}, r_{3}} \circ \Lambda_{s}^{r_{1}, r_{2}}=\frac{\left[\begin{array}{c}
n \\
r_{2}
\end{array}\right]_{q}}{\left[\begin{array}{l}
n \\
s
\end{array}\right]_{q}-\left[\begin{array}{c}
n \\
s-1
\end{array}\right]_{q}} \Lambda_{s}^{r_{1}, r_{3}} .
$$

The proof of Theorem 4.1 requires two preliminary lemmas. In the first one we reduce the proof of the formula $\left[\mathrm{P}_{s}\left(r_{1}, r_{2}, r_{3}\right)\right]$ to two particular cases.

Lemma 4.2. The product formula $\left(\mathrm{P}_{s}\left(r_{1}, r_{2}, r_{3}\right)\right]$ is implied by:

- $\mathrm{P}_{s}\left(r_{1}, r_{2}, r_{3}\right)$ for $r_{1}=r_{3}$.

- $\mathrm{P}_{s}\left(r_{1}, r_{2}, r_{3}\right)$ for $r_{1} \leq r_{2} \leq r_{3}$.

Proof. In what follows we shall write

$$
\mathrm{k}(r, s)=\frac{\left[\begin{array}{l}
n \\
r
\end{array}\right]_{q}}{\left[\begin{array}{l}
n \\
s
\end{array}\right]_{q}-\left[\begin{array}{c}
n \\
s-1
\end{array}\right]_{q}} .
$$

Let $\mathrm{P}(r, s): \mathrm{V}_{r} \rightarrow \mathrm{V}_{r, s}$ be the orthogonal projection. Then, (2) and (6) give

$$
\begin{aligned}
\operatorname{tr}(\mathrm{P}(r, s)) & =\operatorname{dim} \mathrm{V}_{r, s}=\left[\begin{array}{l}
n \\
s
\end{array}\right]_{q}-\left[\begin{array}{c}
n \\
s-1
\end{array}\right]_{q}, \\
\operatorname{tr}\left(\Lambda_{s}^{r, r}\right) & =\sum_{x \in \mathrm{X}_{r}} \lambda_{s}^{r, r}(\partial(x, x))=\left[\begin{array}{l}
n \\
r
\end{array}\right]_{q} .
\end{aligned}
$$

In particular, it is clear that

$$
\mathrm{P}(r, s)=\frac{\left[\begin{array}{l}
n \\
s
\end{array}\right]_{q}-\left[\begin{array}{c}
n \\
s-1
\end{array}\right]_{q}}{\left[\begin{array}{l}
n \\
r
\end{array}\right]_{q}} \Lambda_{s}^{r, r}=\mathrm{k}(r, s)^{-1} \Lambda_{s}^{r, r} .
$$

Assuming $\left[\mathrm{P}_{s}\left(r_{1}, r_{2}, r_{3}\right)\right]$ for $r_{1}=r_{3}$, we claim that

$$
\mathrm{P}_{s}\left(r_{1}, r_{3}, r_{2}\right) \Leftrightarrow \mathrm{P}_{s}\left(r_{1}, r_{2}, r_{3}\right) \Leftrightarrow \mathrm{P}_{s}\left(r_{2}, r_{1}, r_{3}\right) .
$$


For instance, we have

$$
\begin{aligned}
\Lambda_{s}^{r_{3}, r_{2}} \circ \Lambda_{s}^{r_{1}, r_{3}} & =\mathrm{k}\left(r_{2}, s\right)^{-1} \Lambda_{s}^{r_{3}, r_{2}} \circ \Lambda_{s}^{r_{2}, r_{3}} \circ \Lambda_{s}^{r_{1}, r_{2}} \\
& =\mathrm{k}\left(r_{3}, s\right) \mathrm{k}\left(r_{2}, s\right)^{-1} \Lambda_{s}^{r_{2}, r_{2}} \circ \Lambda_{s}^{r_{1}, r_{2}} \\
& =\mathrm{k}\left(r_{3}, s\right) \mathrm{P}\left(r_{2}, s\right) \circ \Lambda_{s}^{r_{1}, r_{2}} \\
& =\mathrm{k}\left(r_{3}, s\right) \Lambda_{s}^{r_{1}, r_{2}} .
\end{aligned}
$$

This proves $\mathrm{P}\left(r_{1}, r_{2}, r_{3}\right) \Rightarrow \mathrm{P}\left(r_{1}, r_{3}, r_{2}\right)$ under the assumption of $\mathrm{P}\left(r_{2}, r_{3}, r_{2}\right)$. The other implications in (18) can be checked in a similar way. On the other hand, since that the transpositions $(a, b, c) \mapsto(a, c, b)$ and $(a, b, c) \mapsto(b, a, c)$ generate all permutations of $(a, b, c)$, it suffices to prove $\left(\mathrm{P}_{s}\left(r_{1}, r_{2}, r_{3}\right)\right]$ in the particular case $r_{1} \leq r_{2} \leq r_{3}$. Therefore, we need only to assume the two cases stated above.

Lemma 4.3. Let $f, g \in \mathcal{M}\left(\mathbb{C}^{*}\right)$ so that $f$ is regular at $q^{-t}$ and $g$ is regular at $q^{-t-1 / 2}$ for all integer $t$. Assume also that one of the sets

$$
\left\{t \in \mathbb{Z} \mid f\left(q^{-t}\right) \neq 0\right\} \quad \text { or } \quad\left\{t \in \mathbb{Z} \mid g\left(q^{-t-1 / 2}\right) \neq 0\right\}
$$

is finite. Then we have the following summation by parts formula

$$
\sum_{t \in \mathbb{Z}} f\left(q^{-t}\right) \mathrm{D} g\left(q^{-t}\right) q^{-t}=-\sum_{t \in \mathbb{Z}+\frac{1}{2}} \mathrm{D} f\left(q^{-t}\right) g\left(q^{-t}\right) q^{-t} .
$$

Proof. We have

$$
\begin{aligned}
\sum_{t \in \mathbb{Z}} f\left(q^{-t}\right) \mathrm{D} g\left(q^{-t}\right) q^{-t} & =\frac{1}{q^{-1 / 2}-q^{1 / 2}} \sum_{t \in \mathbb{Z}} f\left(q^{-t}\right)\left(g\left(q^{-t-1 / 2}\right)-g\left(q^{-t+1 / 2}\right)\right) \\
& =\frac{1}{q^{-1 / 2}-q^{1 / 2}} \sum_{t \in \mathbb{Z}+\frac{1}{2}}\left(f\left(q^{-t+1 / 2}\right)-f\left(q^{-t-1 / 2}\right)\right) g\left(q^{-t}\right) \\
& =-\sum_{t \in \mathbb{Z}+\frac{1}{2}} \mathrm{D} f\left(q^{-t}\right) g\left(q^{-t}\right) q^{-t}
\end{aligned}
$$

Proof of $\left(\overline{\mathrm{P}_{s}\left(r_{1}, r_{2}, r_{3}\right)}\right)$ for $r_{1}=r_{3}$. By Schur lemma, the maps $\Lambda_{s}^{r_{2}, r_{1}} \circ \Lambda_{s}^{r_{1}, r_{2}}$ and $\mathrm{k}\left(r_{2}, s\right) \Lambda_{s}^{r_{1}, r_{1}}$ are proportional. Hence, it suffices to prove that both operators have the same trace. Arguing as in Lemma 4.2 the operator $\Lambda_{s}^{r_{1}, r_{1}}$ has trace

$$
\left[\begin{array}{c}
n \\
r_{1}
\end{array}\right]_{q}
$$

In particular, we need to prove that

$$
\operatorname{tr}\left(\Lambda_{s}^{r_{2}, r_{1}} \circ \Lambda_{s}^{r_{1}, r_{2}}\right)=\frac{\left[\begin{array}{c}
n \\
r_{1}
\end{array}\right]_{q}\left[\begin{array}{c}
n \\
r_{2}
\end{array}\right]_{q}}{\left[\begin{array}{l}
n \\
s
\end{array}\right]_{q}-\left[\begin{array}{c}
n \\
s-1
\end{array}\right]_{q}} .
$$

Let $f_{s}$ be a polynomial of degree $s$

$$
f_{s}(u)=\partial_{s} f_{s} u^{s}+\ldots
$$

where the dots stand for terms of lower degree. The action of $\mathrm{D}$ on the main coefficient of $f_{s}$ is given by

$$
\mathrm{D} f_{s}(u)=\partial_{s} f_{s} \frac{q^{s / 2}-q^{-s / 2}}{q^{1 / 2}-q^{-1 / 2}} u^{s-1}+\ldots
$$


see Section 2.2 of $[9$. The iteration of this formula leads to

$$
\begin{aligned}
\mathrm{D}^{s} f_{s}^{r_{1}, r_{2}} & =\partial_{s} f_{s}^{r_{1}, r_{2}}(-1)^{s} q^{-\frac{1}{2}\left(\begin{array}{l}
s \\
2
\end{array}\right)} \frac{\left(q^{s} ; q^{-1}\right)_{s}}{(q-1)^{s}} \\
& =q^{s\left(r_{2}-r_{1}\right)+\frac{1}{2}\left(\begin{array}{l}
s \\
2
\end{array}\right)} \frac{\left(q^{n-s+1}, q^{s} ; q^{-1}\right)_{s}}{(q-1)^{s}\left(q^{n-r_{1}}, q^{r_{2}} ; q^{-1}\right)_{s}},
\end{aligned}
$$

where the last identity follows from (16). Now we are ready to compute the trace of $\Lambda_{s}^{r_{2}, r_{1}} \circ \Lambda_{s}^{r_{1}, r_{2}}$. We begin by writing this trace in terms of the eigenfunction $f_{s}^{r_{1}, r_{2}}$ and the Rodrigues function $\rho^{r_{1}, r_{2}}$. To that aim, we recall the combinatorial meaning of this function, see Remark 3.12 Then, we use the Rodrigues formula given in (17) after the normalization of the functions $f_{s}^{r_{1}, r_{2}}$.

$$
\begin{aligned}
\operatorname{tr}\left(\Lambda_{s}^{r_{2}, r_{1}} \circ \Lambda_{s}^{r_{1}, r_{2}}\right) & =\sum_{x_{1}, x_{2}} \lambda_{s}^{r_{1}, r_{2}}\left(\partial\left(x_{2}, x_{1}\right)\right) \lambda_{s}^{r_{2}, r_{1}}\left(\partial\left(x_{1}, x_{2}\right)\right) \\
& =\sum_{t \in \mathbb{Z}} \rho^{r_{1}, r_{2}}\left(q^{-t}\right) q^{-t} f_{s}^{r_{1}, r_{2}}\left(q^{-t}\right) f_{s}^{r_{2}, r_{1}}\left(q^{r_{2}-r_{1}-t}\right) \\
& =(-1)^{s} \frac{q^{s\left(r_{2}-r_{1}\right)-\frac{1}{2}\left(\begin{array}{c}
s \\
2
\end{array}\right)}(q-1)^{2}}{\left(q^{n-r_{1}}, q^{r_{2}} ; q^{-1}\right)_{s}} \\
& \times \sum_{t \in \mathbb{Z}} f_{s}^{r_{2}, r_{1}}\left(q^{r_{2}-r_{1}-t}\right) D^{s} \rho_{s}^{r_{1}, r_{2}}\left(q^{-t}\right) q^{-t} .
\end{aligned}
$$

Now recall that

$$
f_{s}^{r_{2}, r_{1}}\left(q^{r_{2}-r_{1}-t}\right)-q^{s\left(r_{2}-r_{1}\right)} f_{s}^{r_{1}, r_{2}}\left(q^{-t}\right)
$$

is a polynomial of degree less that $s$ in $u=q^{-t}$. In particular, Lemma 4.3 gives

$$
\sum_{t \in \mathbb{Z}} f_{s}^{r_{2}, r_{1}}\left(q^{r_{2}-r_{1}-t}\right) \mathrm{D}^{s} \rho_{s}^{r_{1}, r_{2}}\left(q^{-t}\right) q^{-t}=q^{s\left(r_{2}-r_{1}\right)} \sum_{t \in \mathbb{Z}} f_{s}^{r_{2}, r_{1}}\left(q^{-t}\right) \mathrm{D}^{s} \rho_{s}^{r_{1}, r_{2}}\left(q^{-t}\right) q^{-t} .
$$

Then, summation by parts and formulas (15) and (19) give

$$
\begin{aligned}
\operatorname{tr}\left(\Lambda_{s}^{r_{2}, r_{1}} \circ \Lambda_{s}^{r_{1}, r_{2}}\right) & =\frac{q^{2 s\left(r_{2}-r_{1}\right)-\frac{1}{2}\left(\begin{array}{l}
s \\
2
\end{array}\right)(q-1)^{2}}}{\left(q^{n-r_{1}}, q^{r_{2}} ; q^{-1}\right)_{s}} \mathrm{D}^{s} f_{s}^{r_{2}, r_{1}} \sum_{t \in \mathbb{Z}+\frac{s}{2}} \rho_{s}^{r_{1}, r_{2}}\left(q^{-t}\right) q^{-t} \\
& =\frac{q^{s\left(r_{2}-r_{1}\right)}\left(q^{n-s+1}, q^{s} ; q^{-1}\right)_{s}}{\left(q^{r_{1}}, q^{n-r_{1}}, q^{r_{2}}, q^{n-r_{2}} ; q^{-1}\right)_{s}} \sum_{t \in \mathbb{Z}+\frac{s}{2}} \rho_{s}^{r_{1}, r_{2}}\left(q^{-t}\right) q^{-t} \\
& =q^{-s} \frac{\left(q^{n-s+1}, q^{s} ; q^{-1}\right)_{s}}{\left(q^{n} ; q^{-1}\right)_{2 s}}\left[\begin{array}{c}
n \\
r_{1}
\end{array}\right]_{q}\left[\begin{array}{c}
n \\
r_{2}
\end{array}\right]_{q} \\
& =\left[\begin{array}{c}
n \\
r_{1}
\end{array}\right]_{q}\left[\begin{array}{c}
n \\
r_{2}
\end{array}\right]_{q} /\left(\left[\begin{array}{c}
n \\
s
\end{array}\right]_{q}-\left[\begin{array}{c}
n \\
s-1
\end{array}\right]_{q}\right) .
\end{aligned}
$$

Notice that, in the use of (19), we interchange the roles of $r_{1}$ and $r_{2}$.

Proof of $\left(\mathrm{P}_{s}\left(r_{1}, r_{2}, r_{3}\right)\right)$ for $r_{1} \leq r_{2} \leq r_{3}$. Given $0 \leq r_{1} \leq r_{2} \leq n$, we define the Radon transform $\mathcal{R}_{\subset}^{r_{1}, r_{2}}: \mathrm{V}_{r_{1}} \rightarrow \mathrm{V}_{r_{2}}$ as follows

$$
\mathcal{R}_{\subset}^{r_{1}, r_{2}} \varphi\left(x_{2}\right)=\sum_{x_{1} \subset x_{2}} \varphi\left(x_{1}\right) .
$$


The kernel of this operator preserves $\partial$ so that $\mathcal{R}_{\subset}^{r_{1}, r_{2}} \in \operatorname{Hom}_{\mathrm{G}}\left(\mathrm{V}_{r_{1}}, \mathrm{~V}_{r_{2}}\right)$. On the other hand, given $\left(x_{1}, x_{3}\right) \in \mathrm{X}_{r_{1}} \times \mathrm{X}_{r_{2}}$, identity (3) gives

$$
\left|\left\{x_{2} \in \mathrm{X}_{r_{2}} \mid x_{1} \subset x_{2} \subset x_{3}\right\}\right|=\left[\begin{array}{l}
r_{3}-r_{1} \\
r_{2}-r_{1}
\end{array}\right]_{q}=\left[\begin{array}{l}
r_{3} \\
r_{2}
\end{array}\right]_{q}\left[\begin{array}{l}
r_{2} \\
r_{1}
\end{array}\right]_{q} /\left[\begin{array}{l}
r_{3} \\
r_{1}
\end{array}\right]_{q} .
$$

In particular,

$$
\mathcal{R}_{\subset}^{r_{2}, r_{3}} \circ \mathcal{R}_{\subset}^{r_{1}, r_{2}}=\frac{\left[\begin{array}{l}
r_{3} \\
r_{2}
\end{array}\right]_{q}\left[\begin{array}{l}
r_{2} \\
r_{1}
\end{array}\right]_{q}}{\left[\begin{array}{l}
r_{3} \\
r_{1}
\end{array}\right]_{q}} \mathcal{R}_{\subset}^{r_{1}, r_{3}}
$$

Since $\mathcal{R}_{\subset}^{r_{1}, r_{2}} \in \operatorname{Hom}_{\mathrm{G}}\left(\mathrm{V}_{r_{1}}, \mathrm{~V}_{r_{2}}\right)$, we decompose it as

$$
\mathcal{R}_{\subset}^{r_{1}, r_{2}}=\sum_{s=0}^{\mathrm{N}\left(r_{1}, r_{2}\right)} \mathrm{w}_{\mathrm{s}}\left(\mathrm{r}_{1}, \mathrm{r}_{2}\right) \Lambda_{s}^{r_{1}, r_{2}} .
$$

To calculate the coefficients $w_{\mathbf{s}}\left(r_{1}, r_{2}\right)$, we observe from (2)

$$
\operatorname{tr}\left(\Lambda_{s}^{r_{2}, r_{1}} \circ \mathcal{R}_{\subset}^{r_{1}, r_{2}}\right)=\sum_{\left(x_{1}, x_{2}\right): x_{1} \subset x_{2}} \lambda_{s}^{r_{2}, r_{1}}\left(\partial\left(x_{1}, x_{2}\right)\right)=\left[\begin{array}{c}
n \\
r_{2}
\end{array}\right]_{q}\left[\begin{array}{c}
r_{2} \\
r_{1}
\end{array}\right]_{q} .
$$

On the other hand,

$$
\Lambda_{s}^{r_{2}, r_{1}} \circ \mathcal{R}_{\subset}^{r_{1}, r_{2}}=\mathrm{w}_{s}\left(r_{1}, r_{2}\right) \Lambda_{s}^{r_{2}, r_{1}} \circ \Lambda_{s}^{r_{1}, r_{2}} .
$$

Therefore, applying $\mathrm{P}_{s}\left(r_{1}, r_{2}, r_{1}\right)$ we obtain

$$
\operatorname{tr}\left(\Lambda_{s}^{r_{2}, r_{1}} \circ \mathcal{R}_{\subset}^{r_{1}, r_{2}}\right)=\mathrm{w}_{s}\left(r_{1}, r_{2}\right) \frac{\left[\begin{array}{c}
n \\
r_{1}
\end{array}\right]_{q}\left[\begin{array}{c}
n \\
r_{2}
\end{array}\right]_{q}}{\left[\begin{array}{c}
n \\
s
\end{array}\right]_{q}-\left[\begin{array}{c}
n \\
s-1
\end{array}\right]_{q}} .
$$

This result leads to the exact value of $\mathrm{w}_{s}\left(r_{1}, r_{2}\right)$. Then, we can rewrite (20) using identity (21). Although we leave the details to the reader, it is not difficult to check that this gives

$$
\sum_{s=0}^{\mathrm{N}\left(r_{1}, r_{2}\right)} \frac{\left(\left[\begin{array}{l}
n \\
s
\end{array}\right]_{q}-\left[\begin{array}{c}
n \\
s-1
\end{array}\right]_{q}\right)^{2}}{\left[\begin{array}{c}
n \\
r_{2}
\end{array}\right]_{q}} \Lambda_{s}^{r_{2}, r_{3}} \circ \Lambda_{s}^{r_{1}, r_{2}}=\sum_{s=0}^{\mathrm{N}\left(r_{1}, r_{2}\right)}\left(\left[\begin{array}{l}
n \\
s
\end{array}\right]_{q}-\left[\begin{array}{c}
n \\
s-1
\end{array}\right]_{q}\right) \Lambda_{s}^{r_{1}, r_{3}} .
$$

Now, since these operators are mutually orthogonal, we identify coefficients.

Corollary 4.4. The Hilbert-Schmidt norm of $\Lambda_{s}^{r_{1}, r_{2}}$ is

$$
\left\|\Lambda_{s}^{r_{1}, r_{2}}\right\|_{\mathrm{HS}}=\left[q^{s\left(r_{2}-r_{1}\right)} \frac{\left(q^{r_{1}}, q^{n-r_{2}} ; q^{-1}\right)_{s}}{\left(q^{n-r_{1}}, q^{r_{2}} ; q^{-1}\right)_{s}} \frac{\left[\begin{array}{c}
n \\
r_{1}
\end{array}\right]_{q}\left[\begin{array}{c}
n \\
r_{2}
\end{array}\right]_{q}}{\left[\begin{array}{c}
n \\
s
\end{array}\right]_{q}-\left[\begin{array}{c}
n \\
s-1
\end{array}\right]_{q}}\right]^{1 / 2} .
$$

Proof. By Schur lemma, there exists some constant $\mathrm{c}_{s}\left(r_{1}, r_{2}\right)$ with

$$
\left(\Lambda_{s}^{r_{1}, r_{2}}\right)^{*}=\mathrm{c}_{s}\left(r_{1}, r_{2}\right) \Lambda_{s}^{r_{2}, r_{1}} .
$$

If we write this relation in terms of the kernels,

$$
\lambda_{s}^{r_{1}, r_{2}}\left(\partial\left(x_{2}, x_{1}\right)\right)=\mathrm{c}_{s}\left(r_{1}, r_{2}\right) \lambda_{s}^{r_{2}, r_{1}}\left(\partial\left(x_{1}, x_{2}\right)\right) \quad \text { for all } \quad\left(x_{1}, x_{2}\right) \in \mathrm{X}_{r_{1}} \times \mathrm{X}_{r_{2}} .
$$

This is equivalent to

$$
f_{s}^{r_{1}, r_{2}}(u)=\mathrm{c}_{s}\left(r_{1}, r_{2}\right) f_{s}^{r_{2}, r_{1}}\left(q^{r_{2}-r_{1}} u\right) .
$$


Identifying the main coefficients via (16), we obtain the value of $\mathrm{c}_{s}\left(r_{1}, r_{2}\right)$

$$
\left(\Lambda_{s}^{r_{1}, r_{2}}\right)^{*}=q^{s\left(r_{2}-r_{1}\right)} \frac{\left(q^{r_{1}}, q^{n-r_{2}} ; q^{-1}\right)_{s}}{\left(q^{n-r_{1}}, q^{r_{2}} ; q^{-1}\right)_{s}} \Lambda_{s}^{r_{2}, r_{1}}
$$

The given expression for the Hilbert-Schmidt norm arises from Theorem 4.1

Remark 4.5. Our choice of the basis $\mathbf{B}$ follows from the condition

$$
f_{s}^{r_{1}, r_{2}}(1)=\lambda_{s}^{r_{1}, r_{2}}(0)=1 .
$$

This normalization is very natural since in this way the kernels $\lambda_{s}^{r, r}$ become the spherical functions associated to the symmetric space $\mathrm{X}_{r}$. This will be an essential observation in Section [5 However, there exist other natural normalizations for $\Lambda_{s}^{r_{1}, r_{2}}$. For instance, Corollary 4.4 provides a normalization for which the basis $\mathbf{B}$ becomes orthonormal with respect to the Hilbert-Schmidt inner product. Moreover, combining the results obtained so far it is not difficult to provide the normalization for which the basis $\mathbf{B}$ is made up of unitary operators.

\section{AN ALTERNATIVE PROOF FOR THE PRODUCT FORMULA}

In this section we provide an alternative proof of $\left(\mathrm{P}_{s}\left(r_{1}, r_{2}, r_{3}\right)\right]$ which does not use any tool from the theory of classical hypergeometric polynomials. In contrast, the main tools will be the characterization of spherical functions given in Theorem 1.3 and the Radon transforms

$$
\mathcal{R}_{\subset}^{r_{1}, r_{2}}: \mathrm{V}_{r_{1}} \rightarrow \mathrm{V}_{r_{2}}
$$

defined for $0 \leq r_{1} \leq r_{2} \leq n$. Along the proof, we shall obtain some identities for the kernels $\lambda_{s}^{r_{1}, r_{2}}$ which might be of independent interest. Before starting the proof, we recall that Lemma 4.2 does not use any argument from the theory of classical hypergeometric polynomials. In particular, we again reduce the proof of the product formula to the proof of those particular cases.

Remark 5.1. Along the proof, we shall assume by convention that

i) $\left[\begin{array}{l}n \\ r\end{array}\right]_{q}=0$ for any integer $r$ not satisfying $0 \leq r \leq n$.

ii) $\Lambda_{s}^{r_{1}, r_{2}}=0$ for any integer $s$ not satisfying $0 \leq s \leq \mathrm{N}\left(r_{1}, r_{2}\right)$.

5.1. Combinatorial identities. Let us consider a subspace $x$ of $\Omega$ of codimension $\partial(\Omega, x)=t$ and let us fix an integer $0 \leq k \leq n$. Our first aim is to calculate the number of $r$-dimensional subspaces $x_{r} \in \mathrm{X}_{r}$ of $\Omega$ satisfying $\partial\left(x_{r}, x\right)=k$. Clearly, this parameter is invariant under the action of G. In particular, it depends on the codimension of $x$ but not on $x$ itself. Hence we define

$$
\mathrm{M}(n, r, t, k)=\left|\left\{x_{r} \in \mathrm{X}_{r} \mid \partial\left(x_{r}, x\right)=k\right\}\right| .
$$

Lemma 5.2. We have

$$
\mathrm{M}(n, r, t, k)=q^{k(n-t-r+k)}\left[\begin{array}{l}
t \\
k
\end{array}\right]_{q}\left[\begin{array}{l}
n-t \\
r-k
\end{array}\right]_{q} .
$$

Proof. Let

$$
\mathrm{A}=\left\{(z, w) \in \mathrm{X}_{r-k} \times \mathrm{X}_{k} \mid z \subset x, w \cap x=\{0\}\right\} .
$$

Then, we compute the cardinality of $\mathrm{A}$ in two different ways. First we notice that an element $x_{r} \in \mathrm{X}_{r}$ satisfies $\partial\left(x_{r}, x\right)=k$ if and only if it can be written as $x_{r}=z \oplus w$ 
with $(z, w) \in \mathrm{A}$. We have only one possible choice for $z=x_{r} \cap x$ while $w$ is any $k$-dimensional subspace with $x_{r}=z \oplus w$. Then, it follows from (4) that

$$
|\mathrm{A}|=\mathrm{M}(n, r, t, k) q^{k(r-k)} .
$$

On the other hand, we can count first how many possible $z$ 's can we plug in A by applying (2) with $(n-t, r-k)$ instead of $(n, r)$. Then we need to count how many $w$ 's can we plug in A. To that aim we notice that, for any such $w$ the element $y=x \oplus w$ is an $(n-t+k)$-dimensional subspace containing $x$. The number of possible $y$ 's is given by (31). Finally, we need to count how many $w$ 's do we have for a fixed $y$, which is given again by (4). In summary, we find that

$$
|\mathrm{A}|=\left[\begin{array}{l}
n-t \\
r-k
\end{array}\right]_{q}\left[\begin{array}{l}
t \\
k
\end{array}\right]_{q} q^{k(n-t)} .
$$

Combining the expressions obtained so far, we obtain the desired result.

In the following result we use Radon transforms and our formula for $\mathrm{M}(n, r, t, k)$ to obtain some useful relations between the kernels $\lambda_{s}^{r_{1}, r_{2}}$ corresponding to a fixed value of the parameter $s$.

Lemma 5.3. Let $0 \leq r_{1}, r_{2}, r_{3} \leq n$ and $0 \leq s \leq n / 2$. Then, there exist absolute constants $\mathrm{c}_{0}, \mathrm{c}_{1}, \mathrm{c}_{2}, \mathrm{c}_{3}$ and $\mathrm{c}_{4}$ such that for any $t \in \mathrm{I}_{n}\left(r_{1}, r_{3}\right)$, we have:

(a) $\lambda_{s}^{r_{1}, r_{3}}(t)=\mathrm{c}_{0} \lambda_{s}^{r_{3}, r_{1}}\left(r_{1}-r_{3}+t\right)$.

(b) If $r_{2} \leq r_{3}$,

(c) If $r_{1} \leq r_{2}$,

$$
\mathrm{c}_{1} \lambda_{s}^{r_{1}, r_{3}}(t)=\sum_{k} \mathrm{M}\left(r_{3}, r_{2}, t, k\right) \lambda_{s}^{r_{1}, r_{2}}(k)
$$

(d) If $r_{2} \leq r_{1}$,

$$
\mathrm{c}_{2}, \lambda_{s}^{r_{1}, r_{3}}(t)=\sum_{k} \mathrm{M}\left(n-r_{1}, n-r_{2}, t, k\right) \lambda_{s}^{r_{2}, r_{3}}(k) .
$$

(e) If $r_{3} \leq r_{2}$,

$$
\mathrm{c}_{3} \lambda_{s}^{r_{1}, r_{3}}(t)=\sum_{k} \mathrm{M}\left(r_{1}, r_{2}, r_{1}-r_{3}+t, r_{2}-r_{3}+k\right) \lambda_{s}^{r_{2}, r_{3}}(k) .
$$

$$
\mathrm{c}_{4} \lambda_{s}^{r_{1}, r_{3}}(t)=\sum_{k} \mathrm{M}\left(n-r_{3}, n-r_{2}, r_{1}-r_{3}+t, r_{1}-r_{2}+k\right) \lambda_{s}^{r_{1}, r_{2}}(k) .
$$

Proof. By Schur lemma, $\left(\Lambda_{s}^{r_{1}, r_{3}}\right)^{*}=\mathrm{c}_{0} \Lambda_{s}^{r_{3}, r_{1}}$ for some constant $\mathrm{c}_{0}$ independent of the variable $t$. Then, (a) follows from the relation between the corresponding kernels and the identity

$$
\partial\left(x_{1}, x_{3}\right)=r_{1}-r_{3}+\partial\left(x_{3}, x_{1}\right) .
$$

To prove (b) we write (again by Schur lemma)

$$
\mathrm{c}_{1} \Lambda_{s}^{r_{1}, r_{3}}=\mathcal{R}_{\subset}^{r_{2}, r_{3}} \circ \Lambda_{s}^{r_{1}, r_{2}} .
$$

Hence, if $\left(x_{1}, x_{3}\right) \in \mathrm{X}_{r_{1}} \times \mathrm{X}_{r_{3}}$

$$
\begin{aligned}
\mathrm{c}_{1} \lambda_{s}^{r_{1}, r_{3}}\left(\partial\left(x_{3}, x_{1}\right)\right) & =\sum_{x_{2} \subset x_{3}} \lambda_{s}^{r_{1}, r_{2}}\left(\partial\left(x_{2}, x_{1}\right)\right) \\
& =\sum_{k}\left|\left\{x_{2} \in \mathrm{X}_{r_{2}} \mid x_{2} \subset x_{3}, \partial\left(x_{2}, x_{1}\right)=k\right\}\right| \lambda_{s}^{r_{1}, r_{2}}(k) .
\end{aligned}
$$


Then we observe that

$$
\begin{aligned}
\mathrm{M}\left(r_{3}, r_{2}, \partial\left(x_{3}, x_{1}\right), k\right) & =\left|\left\{x_{2} \in \mathrm{X}_{r_{2}} \mid x_{2} \subset x_{3}, \partial\left(x_{2}, x_{1} \cap x_{3}\right)=k\right\}\right| \\
& =\left|\left\{x_{2} \in \mathrm{X}_{r_{2}} \mid x_{2} \subset x_{3}, \partial\left(x_{2}, x_{1}\right)=k\right\}\right| .
\end{aligned}
$$

To prove (c) we write $c_{2} \Lambda_{s}^{r_{1}, r_{3}}=\Lambda_{s}^{r_{2}, r_{3}} \circ \mathcal{R}_{\subset}^{r_{1}, r_{2}}$ for some absolute constant $\mathrm{c}_{2}$. Proceeding as above, this gives

$$
\mathrm{c}_{2} \lambda_{s}^{r_{1}, r_{3}}\left(\partial\left(x_{3}, x_{1}\right)\right)=\sum_{k}\left|\left\{x_{2} \in \mathrm{X}_{r_{2}} \mid x_{1} \subset x_{2}, \partial\left(x_{3}, x_{2}\right)=k\right\}\right| \lambda_{s}^{r_{2}, r_{3}}(k) .
$$

To calculate the coefficient, we work in the dual space $\Omega^{*}$. Let $\mathbb{X}$ stand for the set of linear subspaces of $\Omega^{*}$ and $\mathbb{X}_{r}$ the subset of $r$-dimensional subspaces of $\Omega^{*}$. Then, if $x^{\perp} \in \mathbb{X}$ denotes the annihilator of a subspace $x \in \mathrm{X}$, we have

$\partial\left(x^{\perp}, z^{\perp}\right)=\partial\left(x^{\perp}\right)-\partial\left(x^{\perp} \cap z^{\perp}\right)=\partial\left(x^{\perp}\right)-\partial\left((x+z)^{\perp}\right)=\partial(x+z)-\partial(x)=\partial(z, x)$.

In particular,

$$
\begin{aligned}
\mathrm{M} & \left(n-r_{1}, n-r_{2}, \partial\left(x_{3}, x_{1}\right), k\right) \\
& =\left|\left\{x_{2}^{\perp} \in \mathbb{X}_{n-r_{2}} \mid x_{2}^{\perp} \subset x_{1}^{\perp}, \partial\left(x_{2}^{\perp}, x_{1}^{\perp} \cap x_{3}^{\perp}\right)=k\right\}\right| \\
& =\left|\left\{x_{2}^{\perp} \in \mathbb{X}_{n-r_{2}} \mid x_{2}^{\perp} \subset x_{1}^{\perp}, \partial\left(x_{2}^{\perp}, x_{3}^{\perp}\right)=k\right\}\right| \\
& =\left|\left\{x_{2} \in \mathrm{X}_{r_{2}} \mid x_{1} \subset x_{2}, \partial\left(x_{3}, x_{2}\right)=k\right\}\right| .
\end{aligned}
$$

Finally, (d) follows from (a) and (b) while (e) follows from (a) and (c).

Theorem 5.4. The kernels of the operators in the basis $\mathbf{B}$ satisfy:

(a) If $0 \leq t \leq s$ and $s \leq r \leq n-s$,

$$
\begin{aligned}
\lambda_{s}^{r, s}(t) & =\frac{\left(q^{s-r-1} ; q^{-1}\right)_{t}}{\left(q^{n-r} ; q^{-1}\right)_{t}} \lambda_{s}^{r, s}(0), \\
\lambda_{s}^{n-s, r}(t) & =\frac{\left(q^{r+s-n-1} ; q^{-1}\right)_{t}}{\left(q^{r} ; q^{-1}\right)_{t}} \lambda_{s}^{n-s, r}(0) .
\end{aligned}
$$

In particular, we have $\lambda_{s}^{r, s}(0) \neq 0$ and $\lambda_{s}^{n-s, r}(0) \neq 0$ for all $s \leq r \leq n-s$.

(b) If $0 \leq s \leq \mathrm{N}\left(r_{1}, r_{2}\right)$, there exists a polynomial $f_{s}^{r_{1}, r_{2}}$ of degree $\leq s$ uniquely determined by the condition

$$
f_{s}^{r_{1}, r_{2}}\left(q^{-t}\right)=\lambda_{s}^{r_{1}, r_{2}}(t) \quad \text { for } \quad t \in \mathrm{I}_{n}\left(r_{1}, r_{2}\right) .
$$

Moreover, the degree of $f_{s}^{r_{1}, r_{2}}$ is $s$ and there are non-zero constants $\mathrm{c}_{1}$ and $\mathrm{c}_{2}$ such that

$$
\begin{aligned}
f_{s}^{r_{1}, r_{2}}(u) & =c_{1} \sum_{k=0}^{s} \frac{\left(q^{r_{2}} u, q^{-1}\right)_{s-k}\left(u^{-1} ; q^{-1}\right)_{k} u^{k}}{\left(q^{s-k} ; q^{-1}\right)_{s-k}\left(q^{k} ; q^{-1}\right)_{k}} q^{k\left(r_{2}-s+k\right)} \lambda_{s}^{r_{1}, s}(k), \\
& =c_{2} \sum_{k=0}^{s} \frac{\left(q^{n-r_{1}} u, q^{-1}\right)_{s-k}\left(u^{-1} ; q^{-1}\right)_{k} u^{k}}{\left(q^{s-k} ; q^{-1}\right)_{s-k}\left(q^{k} ; q^{-1}\right)_{k}} q^{k\left(n-r_{1}-s+k\right)} \lambda_{s}^{n-s, r_{2}}(k) .
\end{aligned}
$$

(c) If $0 \leq s \leq \mathrm{N}\left(r_{1}, r_{2}\right)$, there exists a non-zero absolute constant $\mathrm{c}_{0}$ such that

$$
f_{s}^{r_{1}, r_{2}}(u)=c_{0} f_{s}^{r_{2}, r_{1}}\left(q^{r_{2}-r_{1}} u\right) .
$$


(d) If $0 \leq s \leq \mathrm{N}\left(r_{1}, r_{2}\right)$, we have $f_{s}^{r_{1}, r_{2}}(1) \neq 0$ and

$$
\begin{aligned}
\frac{f_{s}^{r_{1}, r_{2}}\left(q^{-r_{2}}\right)}{f_{s}^{r_{1}, r_{2}}(1)} & =\frac{\lambda_{s}^{r_{1}, s}(s)}{\lambda_{s}^{r_{1}, s}(0)}=(-1)^{s} q^{\left(\begin{array}{c}
s \\
2
\end{array}\right)-r_{1} s} \frac{\left(q^{r_{1}} ; q^{-1}\right)_{s}}{\left(q^{n-r_{1}} ; q^{-1}\right)_{s}}, \\
\frac{f_{s}^{r_{1}, r_{2}}\left(q^{r_{1}-n}\right)}{f_{s}^{r_{1}, r_{2}}(1)} & =\frac{\lambda_{s}^{n-s, r_{2}}(s)}{\lambda_{s}^{n-s, r_{2}}(0)}=(-1)^{s} q^{\left(\begin{array}{c}
s \\
2
\end{array}\right)+s\left(r_{2}-n\right)} \frac{\left(q^{n-r_{2}} ; q^{-1}\right)_{s}}{\left(q^{r_{2}} ; q^{-1}\right)_{s}} .
\end{aligned}
$$

In particular, we have

$$
\frac{f_{s}^{r_{1}, r_{2}}\left(q^{r_{1}-r_{2}}\right)}{f_{s}^{r_{1}, r_{2}}(1)}=q^{s\left(r_{2}-r_{1}\right)} \frac{\left(q^{r_{1}}, q^{n-r_{2}} ; q^{-1}\right)_{s}}{\left(q^{n-r_{1}}, q^{r_{2}} ; q^{-1}\right)_{s}} .
$$

(e) If $0 \leq s \leq \mathrm{N}\left(r_{1}, r_{2}\right)$,

$$
\left(\Lambda_{s}^{r_{1}, r_{2}}\right)^{*}=\frac{f_{s}^{r_{1}, r_{2}}(1)}{f_{s}^{r_{2}, r_{1}}(1)} q^{s\left(r_{2}-r_{1}\right)} \frac{\left(q^{r_{1}}, q^{n-r_{2}} ; q^{-1}\right)_{s}}{\left(q^{n-r_{1}}, q^{r_{2}} ; q^{-1}\right)_{s}} \Lambda_{s}^{r_{2}, r_{1}} .
$$

Proof. By Lemma 5.2 and Lemma 5.3 (e) with $\left(r_{1}, r_{2}, r_{3}\right)=(r, s, s-1)$, we have

$$
\begin{aligned}
& \frac{q^{n-r-t}-1}{q-1} q^{r-s+t+1} \lambda_{s}^{r, s}(t+1)+\frac{q^{r-s+t+1}-1}{q-1} \lambda_{s}^{r, s}(t) \\
& =\sum_{k}\left[\begin{array}{c}
r-s+t+1 \\
r-s+k
\end{array}\right]_{q}\left[\begin{array}{c}
n-r-t \\
n-r-k
\end{array}\right]_{q} q^{(r-s+k)(k-t)} \lambda_{s}^{r, s}(k) \\
& =\sum_{k} \mathrm{M}(n-s+1, n-s, r-s+t+1, r-s+k) \lambda_{s}^{r, s}(k) \\
& =\mathrm{c}_{4} \lambda_{s}^{r, s-1}(t)=0 .
\end{aligned}
$$

The first identity in (a) follows by solving the recurrence

$$
\lambda_{s}^{r, s}(t+1)=\frac{1-q^{s-r-t-1}}{1-q^{n-r-t}} \lambda_{s}^{r, s}(t) .
$$

The second identity in (a) follows similarly from Lemma 5.2 and Lemma 5.3 (d) with $\left(r_{1}, r_{2}, r_{3}\right)=(n-s+1, n-s, r)$. For the first identity in (b), we use Lemma 5.2 and Lemma 5.3 (b) with $\left(r_{1}, s, r_{2}\right)$ instead of $\left(r_{1}, r_{2}, r_{3}\right)$

$$
\begin{aligned}
\mathrm{c}_{1} \lambda_{s}^{r_{1}, r_{2}}(t) & =\sum_{k} \mathrm{M}\left(r_{2}, s, t, k\right) \lambda_{s}^{r_{1}, s}(k) \\
& =\sum_{k}\left[\begin{array}{l}
t \\
k
\end{array}\right]_{q}\left[\begin{array}{c}
r_{2}-t \\
s-k
\end{array}\right]_{q} q^{k\left(r_{2}-t-s+k\right)} \lambda_{s}^{r_{1}, s}(k) \\
& =\sum_{k} \frac{\left(q^{r_{2}-t} ; q^{-1}\right)_{s-k}\left(q^{t} ; q^{-1}\right)_{k} q^{-t k}}{\left(q^{s-k} ; q^{-1}\right)_{s-k}\left(q^{k} ; q^{-1}\right)_{k}} q^{k\left(r_{2}-s+k\right)} \lambda_{s}^{r_{1}, s}(k) .
\end{aligned}
$$

Clearly, the right hand side is a polynomial in the variable $u=q^{-t}$ of degree $\leq s$. This gives the first identity in (b) and proves the existence of such a polynomial. Uniqueness follows from $\left|\mathrm{I}_{n}\left(r_{1}, r_{2}\right)\right|=\mathrm{N}\left(r_{1}, r_{2}\right)+1>s$. The second identity in (b) follows in a similar way from Lemma 5.2 and Lemma 5.3 (c) by taking $\left(r_{1}, n-s, r_{2}\right)$ instead of $\left(r_{1}, r_{2}, r_{3}\right)$. From (a) and the first identity in (b), we can write the main coefficient of $f_{s}^{r_{1}, r_{2}}$ as

$$
\sum_{k=0}^{s} \frac{(-1)^{s-k} q^{r_{2} k-\left(\begin{array}{c}
s-k \\
2
\end{array}\right)}}{\left(q^{s-k} ; q^{-1}\right)_{s-k}\left(q^{k} ; q^{-1}\right)_{k}} q^{k\left(r_{2}-s+k\right)} \frac{\left(q^{s-r_{1}-1} ; q^{-1}\right)_{k}}{\left(q^{n-r_{1}} ; q^{-1}\right)_{k}} \lambda_{s}^{r_{1}, s}(0) .
$$


Therefore, since the coefficients of $\lambda_{s}^{r_{1}, s}(0)$ in this sum are all positive (notice that $\left(q^{s-r_{1}-1} ; q^{-1}\right)_{k}$ is positive for all $k \geq 0$ since $\left.s \leq r_{1}\right)$, we deduce that the main coefficient does not vanish so that $f_{s}^{r_{1}, r_{2}}$ has degree $s$. This concludes the proof of (b). Property (c) is another way to write Lemma [5.3 (a). The property $f_{s}^{r_{1}, r_{2}}(1) \neq 0$ follows trivially from (a). The first identity in (d) follows by evaluating the first identity in (b) at $u=q^{-r_{2}}$ and $u=1$ and then applying (a). Similarly, for the second identity in (d), we evaluate the second identity in (b) at $u=q^{r_{1}-n}$ and $u=1$ followed by (a). In both identities, the transformation formula

$$
\left(z ; q^{-1}\right)_{s}=(-1)^{s} q^{-\left(\begin{array}{c}
s \\
2
\end{array}\right) z^{s}\left(q^{s-1} z^{-1} ; q^{-1}\right)_{s}}
$$

is needed. The last identity in (d) follows from the previous ones and (c). To prove the identity in (e), it suffices to notice that the constant $\mathrm{c}_{0}$ in (c) is given by $f_{s}^{r_{1}, r_{2}}(1) / f_{s}^{r_{2}, r_{1}}\left(q^{r_{2}-r_{1}}\right)$ and apply (d). This completes the proof.

5.2. Proof of the cases $r_{1} \leq r_{2} \leq r_{3}$ and $r_{1} \geq r_{2} \geq r_{3}$. We have already seen that $f_{s}^{r_{1}, r_{2}}$ satisfies the condition $f_{s}^{r_{1}, r_{2}}(1) \neq 0$ for any $0 \leq s \leq \mathrm{N}\left(r_{1}, r_{2}\right)$. Hence, from now on we normalize the operators $\Lambda_{s}^{r_{1}, r_{2}}$ in $\mathbf{B}$ requiring $f_{s}^{r_{1}, r_{2}}(1)=1$. In particular, now Theorem 5.4 (e) has the form

$$
\left(\Lambda_{s}^{r_{1}, r_{2}}\right)^{*}=\frac{\mathrm{d}\left(r_{1}, s\right)}{\mathrm{d}\left(r_{2}, s\right)} \Lambda_{s}^{r_{2}, r_{1}} \quad \text { with } \quad \mathrm{d}(r, s)=q^{s r} \frac{\left(q^{n-r} ; q^{-1}\right)_{s}}{\left(q^{r} ; q^{-1}\right)_{s}} .
$$

Let us consider $0 \leq r_{1}, r_{2}, r_{3} \leq n$ with $r_{1} \leq r_{2} \leq r_{3}$. Then we claim that

$$
\mathrm{P}\left(r_{3}, r_{2}, r_{1}\right) \Rightarrow \mathrm{P}\left(r_{1}, r_{2}, r_{3}\right) .
$$

Indeed, let us use the same notation as in Section 4

$$
\mathrm{k}(r, s)=\frac{\left[\begin{array}{l}
n \\
r
\end{array}\right]_{q}}{\left[\begin{array}{l}
n \\
s
\end{array}\right]_{q}-\left[\begin{array}{c}
n \\
s-1
\end{array}\right]_{q}} .
$$

Then (22) gives

$$
\begin{aligned}
\Lambda_{s}^{r_{2}, r_{3}} \circ \Lambda_{s}^{r_{1}, r_{2}} & =\frac{\mathrm{d}\left(r_{3}, s\right)}{\mathrm{d}\left(r_{2}, s\right)}\left(\Lambda_{s}^{r_{3}, r_{2}}\right)^{*} \frac{\mathrm{d}\left(r_{2}, s\right)}{\mathrm{d}\left(r_{1}, s\right)}\left(\Lambda_{s}^{r_{2}, r_{1}}\right)^{*} \\
& =\frac{\mathrm{d}\left(r_{3}, s\right)}{\mathrm{d}\left(r_{1}, s\right)}\left(\Lambda_{s}^{r_{2}, r_{1}} \circ \Lambda_{s}^{r_{3}, r_{2}}\right)^{*} \\
& =\frac{\mathrm{d}\left(r_{3}, s\right)}{\mathrm{d}\left(r_{1}, s\right)} \mathrm{k}\left(r_{2}, s\right)\left(\Lambda_{s}^{r_{3}, r_{1}}\right)^{*}=\mathrm{k}\left(r_{2}, s\right) \Lambda_{s}^{r_{1}, r_{3}} .
\end{aligned}
$$

In summary, it suffices to prove the case $r_{1} \geq r_{2} \geq r_{3}$. Notice that this is clear since Lemma 4.2 obviously holds with $r_{1} \geq r_{2} \geq r_{3}$ instead of $r_{1} \leq r_{2} \leq r_{3}$. However, we have proved the implication $\mathrm{P}\left(r_{3}, r_{2}, r_{1}\right) \Rightarrow \mathrm{P}\left(r_{1}, r_{2}, r_{3}\right)$ since we shall need both results in Paragraph 5.3

Remark 5.5. Let us write again $\mathrm{P}(r, s): \mathrm{V}_{r} \rightarrow \mathrm{V}_{r, s}$ for the orthogonal projection from $\mathrm{V}_{r}$ onto $\mathrm{V}_{r, s}$. Then, using that $\lambda_{s}^{r, r}(0)=f_{s}^{r, r}(1)=1$ and arguing as in the proof of Lemma 4.2 we have

$$
\mathrm{P}(r, s)=\frac{\operatorname{dim} \mathrm{V}_{r, s}}{\left|\mathrm{X}_{r}\right|} \Lambda_{s}^{r, r}=\mathrm{k}(r, s)^{-1} \Lambda_{s}^{r, r} .
$$

In particular, it turns out that

$$
\Lambda_{s}^{r_{1}, r_{2}} \circ \Lambda_{s}^{r_{1}, r_{1}}=\mathrm{k}\left(r_{1}, s\right) \Lambda_{s}^{r_{1}, r_{2}}
$$




$$
\Lambda_{s}^{r_{2}, r_{2}} \circ \Lambda_{s}^{r_{1}, r_{2}}=\mathrm{k}\left(r_{2}, s\right) \Lambda_{s}^{r_{1}, r_{2}} .
$$

That is, $\left.\mathrm{P}_{s}\left(r_{1}, r_{2}, r_{3}\right)\right]$ holds with $r_{1}=r_{2}$ or $r_{2}=r_{3}$. Moreover, recalling the definition of spherical function given in Section 1 and that $\mathrm{X}_{r}$ is a finite symmetric space for any $0 \leq r \leq n$, we observe from (23) that the set of spherical functions associated to $\mathrm{X}_{r}$ is

$$
\left\{\lambda_{s}^{r, r} \mid 0 \leq s \leq r \wedge(n-r)\right\} .
$$

Following Remark [5.5 we are now allowed to use the characterization of spherical functions provided by Theorem 1.3. That is, given $y \in \mathrm{X}_{r}$ for some $0 \leq r \leq n$, we consider the isotropy subgroup of $y$

$$
\mathrm{G}_{y}=\{g \in \mathrm{G} \mid g y=y\} .
$$

Then, Theorem 1.3 gives

$$
\frac{1}{\left|\mathrm{G}_{y}\right|} \sum_{g \in \mathrm{G}_{y}} \lambda_{s}^{r, r}(\partial(g x, z))=\lambda_{s}^{r, r}(\partial(x, y)) \lambda_{s}^{r, r}(\partial(y, z)),
$$

for any $x, y, z \in \mathrm{X}_{r}$. On the other hand, by Remark 5.5 we have

$$
\begin{aligned}
& \lambda_{s}^{r_{1}, r_{2}}\left(\partial\left(x_{2}, x_{1}\right)\right)=\frac{1}{\mathrm{k}\left(r_{1}, s\right)} \sum_{z_{1} \in \mathrm{X}_{r_{1}}} \lambda_{s}^{r_{1}, r_{2}}\left(\partial\left(x_{2}, z_{1}\right)\right) \lambda_{s}^{r_{1}, r_{1}}\left(\partial\left(z_{1}, x_{1}\right)\right), \\
& \lambda_{s}^{r_{1}, r_{2}}\left(\partial\left(x_{2}, x_{1}\right)\right)=\frac{1}{\mathrm{k}\left(r_{2}, s\right)} \sum_{z_{2} \in \mathrm{X}_{r_{2}}} \lambda_{s}^{r_{2}, r_{2}}\left(\partial\left(x_{2}, z_{2}\right)\right) \lambda_{s}^{r_{1}, r_{2}}\left(\partial\left(z_{2}, x_{1}\right)\right) .
\end{aligned}
$$

Combining these identities with (24), we obtain

$$
\begin{aligned}
& \frac{1}{\left|\mathrm{G}_{y_{1}}\right|} \sum_{g \in \mathrm{G}_{y_{1}}} \lambda_{s}^{r_{1}, r_{2}}\left(\partial\left(x_{2}, g x_{1}\right)\right)=\lambda_{s}^{r_{1}, r_{2}}\left(\partial\left(x_{2}, y_{1}\right)\right) \lambda_{s}^{r_{1}, r_{1}}\left(\partial\left(y_{1}, x_{1}\right)\right), \\
& \frac{1}{\left|\mathrm{G}_{y_{2}}\right|} \sum_{g \in \mathrm{G}_{y_{2}}} \lambda_{s}^{r_{1}, r_{2}}\left(\partial\left(g x_{2}, x_{1}\right)\right)=\lambda_{s}^{r_{2}, r_{2}}\left(\partial\left(x_{2}, y_{2}\right)\right) \lambda_{s}^{r_{1}, r_{2}}\left(\partial\left(y_{2}, x_{1}\right)\right) .
\end{aligned}
$$

with $\left(y_{1}, y_{2}\right) \in \mathrm{X}_{r_{1}} \times \mathrm{X}_{r_{2}}$. Now, let us assume that $r_{1} \geq r_{2} \geq r_{3}$ and let us take $x_{k} \in \mathrm{X}_{r_{k}}$ for $k=1,2,3$ with $x_{3} \subset x_{2} \subset x_{1}$. Clearly, for any $g \in \mathrm{G}_{x_{2}}$ we will have $x_{3} \subset x_{2} \subset g x_{1}$ so that $\partial\left(x_{3}, g x_{1}\right)=0$. On the other hand, by Schur lemma we have

$$
\Lambda_{s}^{r_{2}, r_{3}} \circ \Lambda_{s}^{r_{1}, r_{2}}=\mathrm{k}_{s}\left(r_{1}, r_{2}, r_{3}\right) \Lambda_{s}^{r_{1}, r_{3}}
$$

for some constant $\mathrm{k}_{s}\left(r_{1}, r_{2}, r_{3}\right)$. Putting the previous results all together, we have

$$
\begin{aligned}
\mathrm{k}_{s}\left(r_{1}, r_{2}, r_{3}\right) & =\frac{\mathrm{k}_{s}\left(r_{1}, r_{2}, r_{3}\right)}{\left|\mathrm{G}_{x_{2}}\right|} \sum_{g \in \mathrm{G}_{x_{2}}} \lambda_{s}^{r_{1}, r_{3}}\left(\partial\left(x_{3}, g x_{1}\right)\right) \\
& =\frac{1}{\left|\mathrm{G}_{x_{2}}\right|} \sum_{g \in \mathrm{G}_{x_{2}}} \sum_{z_{2} \in \mathrm{X}_{r_{2}}} \lambda_{s}^{r_{2}, r_{3}}\left(\partial\left(x_{3}, z_{2}\right)\right) \lambda_{s}^{r_{1}, r_{2}}\left(\partial\left(z_{2}, g x_{1}\right)\right) \\
& =\frac{1}{\left|\mathrm{G}_{x_{2}}\right|} \sum_{g \in \mathrm{G}_{x_{2}}} \sum_{z_{2} \in \mathrm{X}_{r_{2}}} \lambda_{s}^{r_{2}, r_{3}}\left(\partial\left(x_{3}, z_{2}\right)\right) \lambda_{s}^{r_{1}, r_{2}}\left(\partial\left(g z_{2}, x_{1}\right)\right) \\
& =\sum_{z_{2} \in \mathrm{X}_{r_{2}}} \lambda_{s}^{r_{2}, r_{3}}\left(\partial\left(x_{3}, z_{2}\right)\right) \lambda_{s}^{r_{2}, r_{2}}\left(\partial\left(z_{2}, x_{2}\right)\right) \lambda_{s}^{r_{1}, r_{2}}\left(\partial\left(x_{2}, x_{1}\right)\right) \\
& =\mathrm{k}\left(r_{2}, s\right) \lambda_{s}^{r_{2}, r_{3}}\left(\partial\left(x_{3}, x_{2}\right)\right) \lambda_{s}^{r_{1}, r_{2}}\left(\partial\left(x_{2}, x_{1}\right)\right)=\mathrm{k}\left(r_{2}, s\right) .
\end{aligned}
$$


5.3. Proof of the case $r_{1}=r_{3}$. In this paragraph we shall need to use another type of Radon transforms. Given an integer $0 \leq r \leq n$, we consider the Radon transform $\mathcal{R}_{c}^{r}: \mathrm{V}_{r} \rightarrow \mathrm{V}_{n-r}$ defined as follows

$$
\mathcal{R}_{c}^{r} \varphi(z)=\sum_{x: x \cap z=\{0\}} \varphi(x) \quad \text { for } \quad z \in \mathrm{V}_{n-r} .
$$

Since the kernel of $\mathcal{R}_{c}^{r}$ is invariant under the action of $\mathrm{G}, \mathcal{R}_{c}^{r} \in \operatorname{Hom}_{\mathrm{G}}\left(\mathrm{V}_{r}, \mathrm{~V}_{n-r}\right)$.

Lemma 5.6. If $r_{1}+r_{2} \leq n$, we have $\mathcal{R}_{c}^{r_{2}} \circ \Lambda_{s}^{r_{1}, r_{2}}=\mathrm{m}\left(r_{2}, s\right) \Lambda_{s}^{r_{1}, n-r_{2}}$ with

$$
\mathrm{m}(r, s)=(-1)^{s} q^{(r-s)(n-r)+\left(\begin{array}{c}
s \\
2
\end{array}\right)} \frac{\left(q^{n-r} ; q^{-1}\right)_{s}}{\left(q^{r} ; q^{-1}\right)_{s}} .
$$

Proof. By Schur lemma, we know the existence of a constant c such that

$$
\mathcal{R}_{c}^{r_{2}} \circ \Lambda_{s}^{r_{1}, r_{2}}=\mathrm{c} \Lambda_{s}^{r_{1}, n-r_{2}} .
$$

When $r_{1}+r_{2} \leq n$, we can take $x_{1} \in \mathrm{X}_{r_{1}}$ and $z_{2} \in \mathrm{X}_{n-r_{2}}$ such that $x_{1} \subset z_{2}$. Then, $\partial\left(z_{2}, x_{1}\right)=n-r_{1}-r_{2}$ and identity (4) gives

$$
\begin{aligned}
\mathrm{c} \lambda_{s}^{r_{1}, n-r_{2}}\left(n-r_{1}-r_{2}\right) & =\sum_{x_{2}: x_{2} \cap z_{2}=\{0\}} \lambda_{s}^{r_{1}, r_{2}}\left(\partial\left(x_{2}, x_{1}\right)\right) \\
& =q^{r_{2}\left(n-r_{2}\right)} \lambda_{s}^{r_{1}, r_{2}}\left(r_{2}\right) .
\end{aligned}
$$

Since we have

$$
\begin{aligned}
\lambda_{s}^{r_{1}, r_{2}}\left(r_{2}\right) & =f_{s}^{r_{1}, r_{2}}\left(q^{-r_{2}}\right), \\
\lambda_{s}^{r_{1}, n-r_{2}}\left(n-r_{1}-r_{2}\right) & =f_{s}^{r_{1}, n-r_{2}}\left(q^{r_{1}+r_{2}-n}\right),
\end{aligned}
$$

the desired relation can be easily checked by applying Theorem 5.4 (d).

Now we are ready to complete the proof. Arguing as in Section 4 the product formula

$$
\Lambda_{s}^{r_{2}, r_{1}} \circ \Lambda_{s}^{r_{1}, r_{2}}=\mathrm{k}\left(r_{2}, s\right) \Lambda_{s}^{r_{1}, r_{1}}
$$

is equivalent to

$$
\operatorname{tr}\left(\Lambda_{s}^{r_{2}, r_{1}} \circ \Lambda_{s}^{r_{1}, r_{2}}\right)=\frac{\left[\begin{array}{c}
n \\
r_{1}
\end{array}\right]_{q}\left[\begin{array}{c}
n \\
r_{2}
\end{array}\right]_{q}}{\left[\begin{array}{c}
n \\
s
\end{array}\right]_{q}-\left[\begin{array}{c}
n \\
s-1
\end{array}\right]_{q}} .
$$

Since $\operatorname{tr}\left(\Lambda_{s}^{r_{2}, r_{1}} \circ \Lambda_{s}^{r_{1}, r_{2}}\right)=\operatorname{tr}\left(\Lambda_{s}^{r_{1}, r_{2}} \circ \Lambda_{s}^{r_{2}, r_{1}}\right)$, we assume without lost of generality that $r_{1} \leq r_{2}$. In particular, we have $s \leq r_{1} \leq r_{2} \leq n-s$. Multiplying on the left (resp. right) of (25) by $\Lambda_{s}^{r_{2}, s}$ (resp. $\Lambda_{s}^{s, r_{1}}$ ) and applying the results obtained in Paragraph 5.2] it turns out that the proof of (25) is equivalent to the proof of

$$
\Lambda_{s}^{r_{2}, s} \circ \Lambda_{s}^{s, r_{2}}=\mathrm{k}\left(r_{2}, s\right) \Lambda_{s}^{s, s} .
$$

Now, multiplying on the left by $\mathcal{R}_{c}^{s}$ and applying Lemma[5.6] (notice that $r_{2}+s \leq n$ and $s+s \leq n$ ), the proof of (25) becomes equivalent to

$$
\Lambda_{s}^{r_{2}, n-s} \circ \Lambda_{s}^{s, r_{2}}=\mathrm{k}\left(r_{2}, s\right) \Lambda_{s}^{s, n-s} .
$$

However, this identity holds since $s \leq r_{2} \leq n-s$. Therefore, the proof is completed. 


\section{REFERENCES}

1. G.E. Andrews, R. Askey and R. Roy, Special Functions, Encyclopedia of Mathematics and its Applications 71, Cambridge Univ. Press, 1999.

2. R. Askey and J.A. Wilson, Some basic hypergeometric orthogonal polynomials that generalize Jacobi polynomials, Mem. Amer. Math. Soc. 54 (1985), no. 319.

3. E. Bannai and T. Ito, Algebraic Combinatorics I: Association Schemes, Benjamin/Cummings Publishing Co., Inc., Menlo Park, 1984.

4. A.E. Brouwer, A.M. Cohen and A. Neumaier, Distance regular graphs, Springer-Verlag, 1989.

5. C.F. Dunkl, An addition theorem for some q-Hahn polynomials, Monasth. Math. 85 (1977), 5-37.

6. G. Gasper and M. Rahman, Basic hypergeometric series. With a foreword by Richard Askey., Encyclopedia of Mathematics and its Applications 35, Cambridge Univ. Press, 1990.

7. J.H. van Lint and R.M. Wilson, A course in combinatorics, Cambridge Univ. Press, 1992.

8. J.M. Marco and J. Parcet, On the natural representation of $S(\Omega)$ into $L^{2}(\mathcal{P}(\Omega))$ : discrete harmonics and Fourier transform, J. Combin. Theory Ser. A 100 (2002), 153-175.

9. J.M. Marco and J. Parcet, A new approach to the theory of classical hypergeometric polynomials. To appear in Trans. Amer. Math. Soc.

10. A.F. Nikiforov, S.K. Suslov and V.B. Uvarov, Classical orthogonal polynomials of a discrete variable, Springer Series in Computational Physics, Springer-Verlag, Berlin, 1991.

11. A. Terras, Fourier Analysis on Finite Groups and Applications, London Math. Soc. Stud. Texts, 1999.

Department of Mathematics, Universidad Autónoma de Madrid, Madrid 28049, Spain

E-mail address: javier.parcet@uam.es 